\title{
KSHV Rta promoter specification and viral reactivation
}

\author{
Jonathan Guito ${ }^{1,2}$ and David M. Lukac ${ }^{1,2 *}$ \\ Graduate School of Biomedical Sciences, New Jersey Medical School, University of Medicine and Dentistry of New Jersey, Newark, NJ, USA \\ ${ }^{2}$ Department of Microbiology and Molecular Genetics, New Jersey Medical School, University of Medicine and Dentistry of New Jersey, Newark, NJ, USA
}

\section{Edited by:}

Keiji Ueda, Osaka University Graduate

School of Medicine, Japan

\section{Reviewed by:}

Hiroki Isomura, Aichi Cancer Center

Research Institute, Japan

Ke Lan, Institut Pasteur of

Shanghai - Chinese Academy of

Sciences, China

\section{*Correspondence:}

David M. Lukac, Department of

Microbiology and Molecular Genetics, New Jersey Medical School,

University of Medicine and Dentistry of New Jersey, 225 Warren Street, ICPH E350C, P.O. Box 1709, Newark, NJ 07101, USA.

e-mail: Lukacdm@umdnj.edu
Viruses are obligate intracellular pathogens whose biological success depends upon replication and packaging of viral genomes, and transmission of progeny viruses to new hosts. The biological success of herpesviruses is enhanced by their ability to reproduce their genomes without producing progeny viruses or killing the host cells, a process called latency. Latency permits a herpesvirus to remain undetected in its animal host for decades while maintaining the potential to reactivate, or switch, to a productive life cycle when host conditions are conducive to generating viral progeny. Direct interactions between many host and viral molecules are implicated in controlling herpesviral reactivation, suggesting complex biological networks that control the decision. One viral protein that is necessary and sufficient to switch latent Kaposi's sarcoma-associated herpesvirus (KSHV) into the lytic infection cycle is called K-Rta. K-Rta is a transcriptional activator that specifies promoters by binding DNA directly and interacting with cellular proteins. Among these cellular proteins, binding of K-Rta to RBP-Jk is essential for viral reactivation. In contrast to the canonical model for Notch signaling, RBP-Jk is not uniformly and constitutively bound to the latent KSHV genome, but rather is recruited to DNA by interactions with K-Rta. Stimulation of RBP-Jk DNA binding requires high affinity binding of Rta to repetitive and palindromic "CANT DNA repeats" in promoters, and formation of ternary complexes with RBP-Jk. However, while K-Rta expression is necessary for initiating KSHV reactivation, K-Rta's role as the switch is inefficient. Many factors modulate K-Rta's function, suggesting that KSHV reactivation can be significantly regulated post-Rta expression and challenging the notion that herpesviral reactivation is bistable. This review analyzes rapidly evolving research on KSHV K-Rta to consider the role of K-Rta promoter specification in regulating the progression of KSHV reactivation.

Keywords: KSHV, herpesvirus, reactivation, DNA/protein interactions, DNA binding, RBP-Jk, Rta

\section{INTRODUCTION}

Kaposi's sarcoma-associated herpesvirus (KSHV) is one of five DNA tumor viruses implicated in the etiology of human cancers. The study of small DNA tumor viruses has established a paradigm for viral cancers, which result from non-productive, dead-end infections. For example, in human papillomavirus (HPV) infection, extrachromosomal genomes replicate to produce mature progeny viruses (a productive infection), leading to the formation of benign warts. However, if HPV integrates into the host genome, it chronically expresses its oncogenic early proteins, fails to replicate (a non-productive infection), and elicits cervical cancer (Moody and Laimins, 2010). This binary relationship between alternative outcomes of infection and cell transformation in human infections has received further support as a fundamental oncogenesis mechanism from studies of the recently discovered Merkel cell polyomavirus (Chang and Moore, 2011).

A basic difference between the small and large DNA tumor viruses is the relationship between productive and non-productive infections. While the small DNA tumor viruses transform human cells due to non-productive, inescapably dead-end infections, large DNA tumor viruses establish non-productive infections that are reversible. These large DNA tumor viruses,
KSHV and Epstein-Barr virus (EBV), belong to the family Herpesviridae, and both replicate with latent/reactivating (i.e., non-productive/productive) patterns of infection. In natural infections of humans, both KSHV and EBV establish latency in B cells and are associated with B lymphoid hyperproliferations and cancers (Ganem, 2007; Rickinson and Kieff, 2007). Reactivation of KSHV from latency leads to increased risk of developing Kaposi's sarcoma (KS) and primary effusion lymphoma (PEL). KS tumors develop following KSHV reactivation; in the KS tumors, the virus is found in endothelial cells.

Dramatic advances in understanding the relationship between EBV infection and mechanisms of pathogenesis have been facilitated by the ease with which EBV infects primary B cells de novo. In that model, genetic studies established a strict interdependence between EBV latency and B cell transformation, and led to definition of the minimal EBV genome sufficient for that effect (Kieff and Rickinson, 2007). Similar progress in understanding KSHV biology has been hampered by the difficulty in establishing robust models of de novo infection that support long-lived viral persistence and transformation. Nonetheless, the models that have been most revealing suggest a remarkable contribution of productive cycle genes to cellular survival and growth control. Most of these 
viral genes are homologous to human genes (Russo et al., 1996). Mechanisms that control expression of the productive cycle genes and progression of the viral reactivation program are thus keys to understanding cellular growth control during KSHV infection.

\section{PEL CELL MODEL OF KSHV LATENCY AND REACTIVATION}

Although long-term latency in tissue culture is not established following de novo KSHV infection (Renne et al., 1998; Grundhoff and Ganem, 2004), latently infected B cells can be explanted and cultured from human afflicted with PEL (Cesarman et al., 1995; Moore et al., 1996; Renne et al., 1996; Boshoff et al., 1998; Drexler et al., 1998). These PEL cell lines maintain KSHV latency for multiple passages, supporting very limited viral gene expression and little virus production (Renne et al., 1996). Treatment of these cells with the protein kinase $\mathrm{C}$-activator 12-O-tetradecanoyl phorbol-13-acetate (TPA) induces a dramatic increase in the number of viral genes transcribed, and release of viral progeny (Renne et al., 1996). Since most of the TPA-induced transcripts encode homologs of productive cycle genes from other herpesviruses (Sarid et al., 1998), these data established PEL cells as authentic models for KSHV latency and reactivation.

Samples derived from KS tissue show transcription patterns similar to un-treated PEL cells (Zhong et al., 1996; Sun et al., 1999) most infected endothelial cells express latent stage transcripts, but $1-10 \%$ of cells express productive cycle transcripts (Staskus et al., 1997, 1999; Sun et al., 1999). Most of the viral homologs of cellular genes are expressed with a productive cycle transcription pattern (reviewed in Mesri et al., 2010).

The development of KSHV microarrays permitted initial kinetic classification of the viral transcriptome. Less than $10 \mathrm{KSHV}$ genes are expressed during latency in PEL cells, but most viral genes are induced following reactivation. With a few exceptions, the viral mRNAs that encode homologs of cellular proteins are expressed with kinetics similar to that of the viral transcripts for DNA replication proteins (Jenner et al., 2001; Paulose-Murphy et al., 2001). Most of the viral transcripts that encode structural proteins are expressed following the transcripts for the replication proteins (Jenner et al., 2001; Paulose-Murphy et al., 2001).

Formal definition of stage-specific transcription was established using microarrays and approaches validated for other herpesviruses. Four of the latent transcripts are expressed constitutively in both latency and reactivation, while all other transcripts are induced during reactivation (Fakhari and Dittmer, 2002). Eighteen miRNAs are also expressed during KSHV latency, and further induced during reactivation (Cai et al., 2005; Samols et al., 2005). Nine transcripts were defined as immediate early (IE) based on their expression in the absence of de novo protein synthesis (Zhu et al., 1999; Rimessi et al., 2001). Twenty-seven transcripts were defined as late (L) based on their sensitivity to treatment with a viral replication inhibitor (Lu et al., 2004). By default classification, 42 transcripts were expressed with delayed early (DE) kinetics, filling the temporal class that follows IE expression but precedes viral DNA replication. As in the other herpesviruses, the existence of distinct classes of transcription in KSHV implies the existence of mechanisms that regulate gene-specific transactivation.

Recent studies used tiling microarrays and limiting dilution quantitative-reverse-transcription (qRT)-PCR to further define stage-specific transcription. These studies showed that a few transcripts commonly classified as lytic are latently expressed in selected cells or conditions (Chandriani and Ganem, 2010). Following reactivation, nearly the entire KSHV genome, including both DNA strands, is transcribed to high levels (Chandriani et al., 2010; Xu and Ganem, 2010). These analyses identified a complex set of previously un-appreciated and abundant productive cycle transcripts. Many of these transcripts are very large, and are expressed in the anti-sense orientation to coding transcripts and miRNAs.

\section{Rta IS THE KSHV LYTIC SWITCH PROTEIN Rta IS SUFFICIENT TO REACTIVATE KSHV FROM LATENCY}

A series of studies established that a single KSHV gene called Rta encoded the only viral protein both necessary and sufficient to reactivate the virus from latency.

Publication of the complete KSHV genome sequence and the demonstration of its extensive homology and collinearity with EBV (Russo et al., 1996) permitted identification of candidate KSHV lytic switch proteins. KSHV encodes two homologs of the EBV transactivating switches Rta ("E-Rta") and Zta in open reading frames (ORFs) 50 and K8, respectively (Sun et al., 1998). In $\mathrm{KSHV}$, these proteins are called K-Rta and K-bZIP. Transient transfection of vectors that expressed K-Rta in BC-1 PEL cells induced the lytic cycle transcripts PAN (also known as nut-1), K-bZIP, small viral capsid antigen (sVCA), viral interleukin (vIL)-6, and viral macrophage inflammatory protein (vMIP)-II (Sun et al., 1998). Conversely, transfection of a K-bZIP expression vector failed to induce PAN and vIL-6, demonstrating that K-bZIP is not a functional equivalent of EBV Zta (Sun et al., 1998). The amount of PAN expressed was dependent on the input dose of the K-Rta expression vector. vIL-6 and PAN were induced by K-Rta with DE kinetics, similar to induction by reactivating chemicals (Sun et al., 1998). As sVCA expression required viral genome replication (i.e., sVCA is a true late gene), its induction by Rta suggested that Rta could drive the lytic gene expression cascade to completion. Interestingly, sVCA was induced in only $20 \%$ of the Rta vector-transfected cells.

Transcription of K-Rta was induced by reactivating chemicals prior to detection of the vIL- 6 and K-bZIP transcripts, proving that K-Rta was upstream of those genes in the reactivation cascade (Sun et al., 1998). Truncation at amino acid (aa) 134 eliminated K-Rta's ability to induce lytic cycle gene expression.

Candidate lytic switch proteins were also selected based on early expression during KSHV reactivation in BCBL-1 PEL cells and by homology to EBV transactivators (Lukac et al., 1998). KSHV ORFs 50 (K-Rta), 57, K1, K3, and K5 were transcribed within $6 \mathrm{~h}$ following TPA treatment of BCBL-1 cells, prior to expression of suspected DE genes. Moreover, ORF 57 encoded a homolog of the EBV transactivator Mta. Transfection of a vector expressing K-Rta, but not vectors expressing ORF 57, K1, K3, or K5, induced expression of the ORF 59 and K8.1 proteins from the latent viral genome in BCBL-1 cells (Lukac et al., 1998). Induction of these productive cycle proteins by ectopic K-Rta was quantitatively similar to reactivation stimulated by TPA. Induction of the late protein K8.1 by K-Rta was sensitive to a viral replication inhibitor, agreeing with the conclusion that Rta expression was 
sufficient to reactivate the complete lytic cycle of KSHV. Remarkably, K8.1 expression occurred in only $6.9 \%$ of Rta expressing cells (Lukac et al., 1998).

Despite the identification of K-Rta as the KSHV lytic switch protein, two characteristics of PEL cell lines have hampered molecular studies of KSHV reactivation: PEL cells are poorly transfectable, and undergo low-level spontaneous reactivation under normal culture conditions. Isolation of the HH-B2 PEL cell line overcame these obstacles by permitting demonstration that ectopic expression of K-Rta induced release of encapsidated viral DNA to the culture supernatant (Gradoville et al., 2000). These data formally proved that K-Rta could activate the entire KSHV productive reactivation cycle (Gradoville et al., 2000). More recent studies showed that K-Rta is also sufficient to reactivate the complete productive cycle of KSHV in infected endothelial, $\mathrm{CV}-1$, human fibroblastic, and 293 cells (Bechtel et al., 2003; An et al., 2006).

\section{Rta IS A PHOSPHORYLATED, TRANSCRIPTIONAL TRANSACTIVATOR}

Analyses of ORF 50's RNA and protein products enhanced understanding of K-Rta's role in viral reactivation. K-Rta's aa sequence suggested that it was a transcription factor, since it contains an $\mathrm{N}$-terminal basic domain and a C-terminal acidic domain conserved with E-Rta (Figure 1; Lukac et al., 1998). In E-Rta, the domains mediate DNA binding and dimerization, and transactivation, respectively. K-Rta also contained two putative nuclear localization sequences (NLSs; Figure 1), and a K-Rta-specific polyclonal antiserum confirmed that K-Rta was localized to the nuclei of TPA-treated BCBL-1 cells and transfected CV-1 cells (Lukac et al., 1998). Co-transfection of uninfected CV-1 cells with the K-Rta expression vector and reporter plasmids demonstrated that Rta transactivated the promoters for PAN, thymidine kinase (TK), kaposin, single stranded DNA binding protein (DBP), and DNA polymerase, but not the assembly protein (AP; Lukac et al., 1998). K-Rta transactivated the PAN, TK, and DBP promoters in

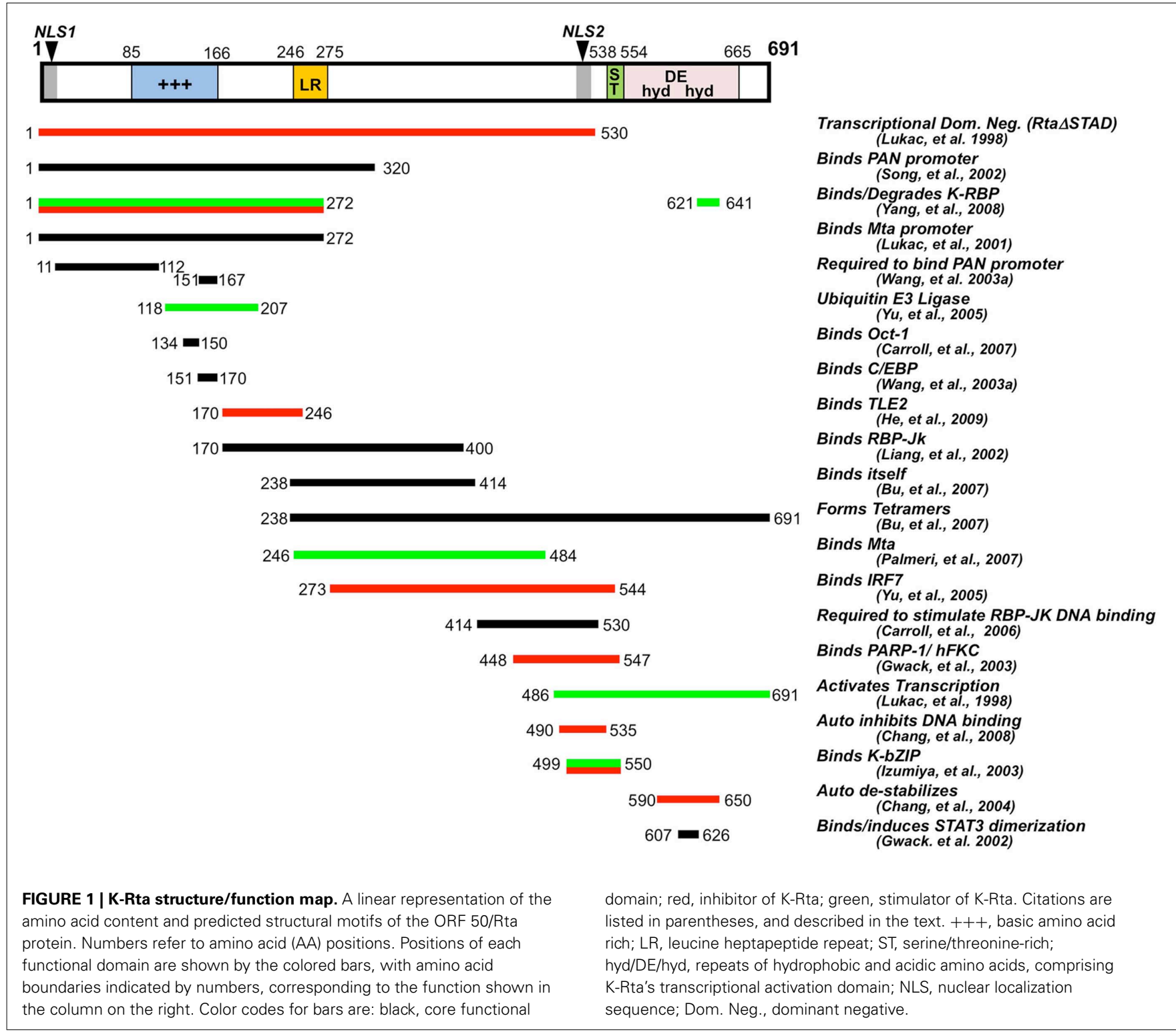


both B cells and endothelial cells (Lukac et al., 1999). These data established that Rta was a direct transactivator of specific viral promoters in the absence of any other viral genes (Lukac et al., 1999).

Northern blotting more clearly distinguished the relative kinetics of K-Rta transcription and that of other early proteins. Abundant Rta expression was detectable within $1 \mathrm{~h}$ post-TPA addition to PEL cells, and preceded detection of transcripts for Mta, K-bZIP, K3, K5, and DBP (Lukac et al., 1999). The ORF 50 locus expresses multiple, divergent transcripts, but only a single, 3,402 nt transcript encodes the sense K-Rta sequence (Lukac et al., 1999). This major transcript initiates upstream of ORF 49, is spliced to an acceptor site upstream of ORF 50, and then continues un-spliced through ORF 50. Downstream of ORF 50, the transcript is spliced three additional times, and encodes K-bZIP and K8.1. The major protein expressed from ORF 50 during reactivation is approximately $110 \mathrm{kDa}$, about $36 \mathrm{kDa}$ greater than the predicted molecular mass of $73.7 \mathrm{kDa}$ (Lukac et al., 1999). The $110-\mathrm{kDa}$ protein is also detected in Cos cells transfected with an ORF 50 expression vector. A second, minor protein of $90 \mathrm{kDa}$ is also detected by the K-Rta antiserum in TPA-induced PEL cell extracts, and migrates identically to the protein produced by programming rabbit reticulocyte lysates (RRL) with the ORF 50 cDNA (Lukac et al., 1999). Phosphatase treatment of the $110-\mathrm{kDa}$ K-Rta protein decreased its abundance and increased accumulation of the $90-\mathrm{kDa}$ species, suggesting that $20 \mathrm{kDa}$ of K-Rta's apparent MW is attributable to phosphorylation (Lukac et al., 1999). Thus, K-Rta has the potential to be regulated by post-translational modification.

\section{Rta IS NECESSARY FOR KSHV REACTIVATION}

Ectopic expression of K-Rta in PEL cells had established that K-Rta was sufficient to induce the full lytic cycle of KSHV gene expression. Structure-function studies of K-Rta led to experiments that proved that K-Rta was also necessary for KSHV reactivation. KRta's transcriptional activation domain was identified by fusing full-length and truncated mutants of K-Rta to the DNA binding domain of the yeast protein Gal4. Among these truncations, only K-Rta aa 486-691 were sufficient to activate transcription of a reporter gene containing binding sites specific for Gal4, in B and endothelial cells (Lukac et al., 1999). These data indicated that K-Rta's activation domain is contained within its C-terminal 206 aa (Figure 1; Lukac et al., 1998; Seaman et al., 1999; Wang et al., 2001a). This sequence is homologous to the activation domains of many cellular and viral transcription factors (Lukac et al., 1999). Deletion of the C-terminal 162 aa from wild type, cognate K-Rta generated a mutant called Rta $\triangle$ STAD (also known as ORF50 $\Delta$ STAD; Figure 1), that was unable to transactivate KSHV promoters or reactivate the virus from latency in PEL cells (Lukac et al., 1999). These data proved that transcriptional activation by K-Rta was required for KSHV reactivation. Furthermore, this Rta $\triangle$ STAD bound to WT K-Rta and specifically inhibited K-Rtamediated transactivation. Proving that K-Rta was necessary for KSHV reactivation, Rta $\triangle S T A D$ blocked viral reactivation that was either spontaneous or induced by TPA or sodium butyrate in PEL cells (Lukac et al., 1999).

Formal genetic proof that K-Rta is required for KSHV reactivation came from studies of a bacmid clone of KSHV in which
ORF 50 was deleted (called BAC36 $\Delta 50$ ). Viral infection was established by transfecting 293 cells with BAC36 50 DNA and selecting with hygromycin. TPA failed to reactivate BAC $36 \Delta 50$, as measured by DNA replication, production of infectious virus in cell supernatants, or expression of K-bZIP, ORF 40/41, K8.1, ORF 57, or ORF 59 (Xu et al., 2005). Expression of K-Rta in trans rescued reactivation of BAC $36 \Delta 50$, implying the deletion of ORF 50 was the only mutation that contributed to the reactivation defect. In addition, a bacmid clone constitutively expressing Rta (called "KSHV-lyt") entered the productive replication cycle by default upon infecting epithelial, endothelial, and Vero cells (Budt et al., 2011).

\section{Rta FUNCTIONS AS A TETRAMER}

The ability of the Rta mutant Rta $\triangle$ STAD to inhibit KSHV reactivation suggested that Rta must form multimers to reactivate KSHV from latency. Blue Native Polyacrylamide electrophoresis (BN-PAGE) and size exclusion chromatography showed that full-length Rta formed tetramers and decamers in solution (Bu et al., 2007). Deletion of Rta's N-terminal leucine heptapeptide repeat (Rta $\triangle$ LR; aa 244-275; Figure 1) inhibited it's transactivation of the K-bZIP and PAN promoters without affecting its nuclear localization. The $\triangle \mathrm{LR}$ mutation also did not inhibit DNA binding of K-Rta, but altered the pattern of K-Rta/DNA complexes observed in EMSA. In the context of the dominant negative mutant Rta $\triangle$ STAD, deletion of the LR (Rta $\triangle$ STAD $\Delta L R$ ) inhibited its ability to interact with full-length K-Rta and to block K-Rta-mediated transactivation and reactivation. However, the LR alone was not sufficient for K-Rta self-interaction, but required the entire central portion of K-Rta (aa 245-414; Figure 1; Bu et al., 2007).

Despite encoding four leucines spaced at 7 aa intervals, K-Rta's LR is not predicted to form leucine-zipper coiled-coils because it also contains five proline residues (Bu et al., 2007). In fact, the proline content, but not the leucine content, of the LR is critical for determining the oligomeric state of Rta. Mutation of three leucines to prolines in the center of the LR generated a K-Rta mutant (Rta-L3P) that exclusively formed tetramers and retained the ability to transactivate and reactivate the virus (Bu et al., 2007). Mutants of Rta that were unable to form tetramers but retained the ability to form higher-order multimers were reduced in function or were non-functional (Bu et al., 2007). Monomers were never detected with WT or mutant K-Rta. In the context of Rta $\triangle$ STAD, the L3P mutant (Rta $\triangle$ STAD-L3P) retained the ability to bind and dominant-negatively inhibit WT K-Rta (Bu et al., 2007). As differences in Rta multimerization had dramatic effects on its function, the data suggested that Rta's multimerization state is a prime candidate for regulating $\mathrm{KSHV}$ reactivation. The data also suggest that many phenotypes attributed to K-Rta LR mutations could affect both interactions with heterologous proteins and K-Rta homo-oligomerization.

\section{Rta SPECIFIES PROMOTERS FOR TRANSACTIVATION BY BINDING DNA DIRECTLY AND INTERACTING WITH CELLULAR DNA BINDING PROTEINS}

The molecular interactions between host and virus that determine transactivation specificity are critical for understanding DNA virus persistence and replication. Identification of K-Rta as KSHV's lytic switch (Lukac et al., 1998; Sun et al., 1998), and demonstration of 
K-Rta's promoter selectivity (Lukac et al., 1998), led immediately to the question of how Rta specified promoters for transactivation. Intense investigations of Rta specificity have focused on the PAN and Mta promoters as models. Experimental approaches have followed the classic strategy of comparing K-Rta DNA binding specificity with K-Rta transactivation specificity. Studies of PAN have revealed a largely concordant relationship between K-Rta DNA binding and transactivation. Studies of Mta have revealed a more complex relationship between K-Rta DNA binding and interactions with cellular DBPs. Taken together, the PAN and Mta models support the existence of two apparently distinct mechanisms for K-Rta promoter specification. These mechanisms are distinguished by K-Rta's relative dependence on the cellular protein RBP-Jk, and the relative lengths of K-Rta's binding sites. K-Rta promoter specification is further influenced by cell type. Controversies regarding K-Rta promoter specification might be attributable to variations in experimental systems, and are discussed in the Section "Conclusion and Perspectives."

\section{RBP-Jk INDEPENDENT PROMOTERS}

PAN is a non-coding, poly-adenylated nuclear RNA that is the most abundant transcript expressed by reactivating KSHV (Sun et al., 1996; Zhong et al., 1996); recent data shows PAN RNA is essential for viral late gene expression (Borah et al., 2011). The PAN locus from -2974 to +1136 was cloned into a pBluescript vector and transfected into COS-1 and 293T cells (Song et al., 2001). The PAN RNA was undetectable by Northern blotting unless a K-Rta expression vector was co-transfected (Song et al., 2001). Deletion of the promoter to -69 had little effect on K-Rta transactivation, while further deletion to -38 eliminated transactivation (Song et al., 2001). Deletion of the element in the cognate PAN promoter eliminated K-Rta transactivation (Kirshner et al., 2000; Song et al., 2001), and the sequence from -69 to -38 conferred orientation independent Rta responsiveness on a heterologous promoter (Song et al., 2001).

Recombinant, FLAG-tagged, full-length K-Rta, expressed and purified from E. coli, bound to PAN promoter DNA -70/-42 in EMSA, providing a direct correspondence between DNA binding and transactivation (Song et al., 2001). Truncated K-Rta (aa 1-320; Figure 1), expressed and purified from bacteria, bound to the PAN $-78 /-37$ element with high affinity [a dissociation constant $\left(K_{\mathrm{D}}\right)$ of 7.9-8.6 nM; Song et al., 2002]. The minimal DNA sequence for detectable K-Rta binding was $-74 /-45$, and methylation between positions $-67 /-52$ interfered with DNA binding (Figure 2; Song et al., 2002). The extra $14 \mathrm{bp}$ flanking the methylation sensitive sequences were A/T-rich. K-Rta made in RRL, requires aa 11-112 and 151-167 to bind the PAN promoter (Figure 1; Wang et al., 2003a).

Analyses of an extensive set of mutations across positions $-75 /-38$ of the PAN promoter showed a general correspondence between K-Rta binding and transactivation, with some discrepancies (i.e., incomplete concordance between a mutation's effect on binding with its effect on transactivation; Song et al., 2002). Due to Rta's high binding affinity to the relatively short segment of the PAN promoter, it was concluded that Rta binding to the promoter is necessary and sufficient to activate transcription of PAN (Song et al., 2002).

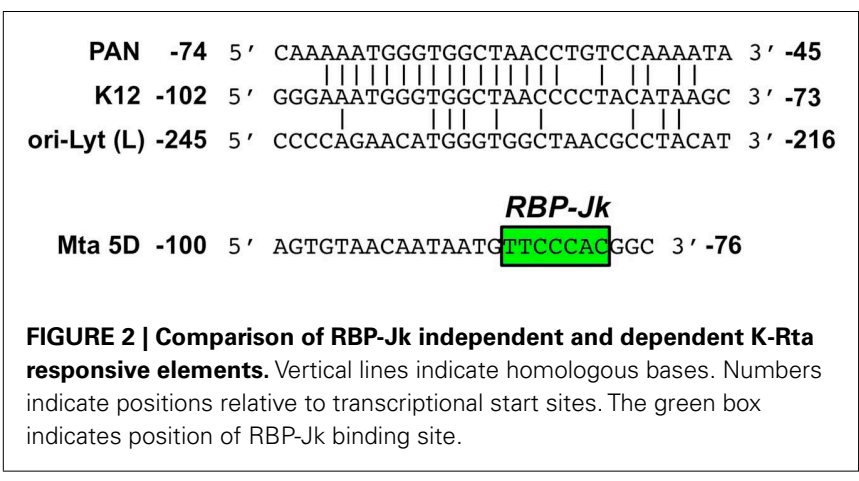

Comparison of the K-Rta responsive element from PAN to other KSHV promoters revealed a 26-bp element from the kaposin (K12) promoter that contained 20 bp homologous to PAN (Figure 2; Song et al., 2002; Chang et al., 2005c). Recombinant K-Rta, cloned as a fusion of aa 1-490 to the VP16 activation domain and expressed in cultured cells, bound to the PAN and kaposin promoters (Chang et al., 2002). The same fusion protein was competent to activate transcription of PAN and kaposin from the latent viral genome in PEL cells (Chang et al., 2002). Mutations in the kaposin element also demonstrated a strict correlation between DNA binding by the K-Rta1-490/VP16 and transactivation (Chang et al., 2002). Further C-terminal truncation of K-Rta to aa 390 was sufficient to bind and transactivate the PAN and kaposin promoters (Chang and Miller, 2004).

The K-Rta binding site from kaposin is also homologous to K-Rta binding sites in the lytic origins of replication (ori-Lyt; Figure 2). K-Rta activates transcription from those sites to participate in lytic replication (AuCoin et al., 2004; Wang et al., 2004b).

\section{RBP-Jk-DEPENDENT PROMOTERS}

In transient transfections of CV-1 cells with a series of KSHV promoters, K-Rta most robustly transactivated those from the ORF 57 and K-bZIP genes (Lukac et al., 1998). Deletion analysis of the ORF 57 promoter revealed that the element critical for Rta transactivation was located between bps -106 and -54 relative to Mta's transcriptional start site (Lukac et al., 2001). While not required for K-Rta transactivation, the promoter sequence between -218 and -106 also contributed positively to transactivation (Lukac et al., 2001). Fusion of the $-106 /-54$ element to a heterologous TATA box conferred K-Rta responsiveness in an orientation independent manner (Lukac et al., 2001).

Initial studies of K-Rta's interaction with the Mta promoter utilized recombinant K-Rta protein that was partially purified from Sf9 cells that were infected with a K-Rta expressing baculovirus. This protein preparation formed three K-Rta-containing complexes with short, overlapping DNAs that spanned the $-106 /-54$ element (Lukac et al., 2001). The DNAs were cloned as dimers in a heterologous reporter vector, and tested for transactivation by K-Rta in CV-1 cells. K-Rta transactivation corresponded with formation of two of the three K-Rta-containing protein/DNA complexes (Lukac et al., 2001). A 26-bp sequence, called Mta 5D (-100/-76; Figure 2), was optimal for K-Rta complex DNA 
binding and transactivation (Lukac et al., 2001). Mta 5D contains a 12-bp, partially palindromic sequence that is conserved with the TATA proximal promoter of the K-bZIP gene. Mutations that inhibited DNA binding of the two K-Rta-containing complexes also eliminated K-Rta transactivation of the Mta and K-bZIP promoters (Lukac et al., 2001).

Site-directed mutagenesis of the Mta 5D element revealed nucleotide-specific variability in K-Rta transactivation and DNA binding (Lukac et al., 2001). Mutations in the upstream side of the $5 \mathrm{D}$ palindrome reduced K-Rta transactivation $50-80 \%$ and reduced, but did not eliminate, DNA binding of the K-Rtacontaining complexes. Mutations in the downstream side of the palindrome eliminated K-Rta transactivation and DNA binding. Later studies suggested that the downstream side of the 5D element was a binding site for K-Rta complexed with the cellular protein Recombination Signal Binding Protein (RBP)-Jk (Figure 2), which co-purified from Sf9 cells (see next paragraph). Truncations of K-Rta, expressed and fully purified from E. coli, demonstrated that K-Rta bound directly to the 5D element, and that K-Rta's DNA binding domain is encoded within aa 1-272 (Lukac et al., 2001).

A two-hybrid screen identified RBP-Jk as a binding partner for K-Rta, and provided a crucial insight into how K-Rta's specifies a sub-set of promoters for transactivation (Liang et al., 2002). RBP-Jk is a nuclear, DBP that specifies Notch-responsive promoters in the canonical model for the Notch signal transduction pathway (Tanigaki and Honjo, 2010). RBP-Jk is essential for KSHV reactivation (Liang and Ganem, 2003). K-Rta and RBP-Jk co-immunoprecipitated from reactivating PEL cells and from cells co-transfected with expression vectors for both proteins (Liang et al., 2002). K-Rta and RBP-Jk also interacted in GST pull-down assays, which identified two independent K-Rta-interacting domains in RBP-Jk, aa 1-180 and 179-360 (Liang et al., 2002). Similar experiments identified the smallest fragment of K-Rta that interacted with RBP-Jk as aa $170-400$ (Figure 1).

The significance of RBP-Jk in K-Rta promoter specification was shown by transient transfection-reporter assays. K-Rta transactivated artificial promoters containing multimerized WT, but not mutant, RBP-Jk binding sites, in an orientation independent manner (Liang et al., 2002). K-Rta failed to transactivate these promoters in transfected mouse embryonic fibroblasts (MEFs) that were genetically RBP-Jk null (OT-11 cells), unless RBP-Jk was ectopically co-expressed (Liang et al., 2002).

Similar to the right side of the Mta promoter 5D element (Figure 2), the KSHV DBP promoter also contains a consensus RBP-Jk site (Liang et al., 2002). However, the PAN promoter does not contain an obvious RBP-Jk site. Rta transactivation of the Mta and DBP promoters, but not the PAN promoter, was eliminated in OT-11 cells, but rescued by ectopic RBP-Jk expression. Mutation of the RBP-Jk sites eliminated K-Rta transactivation of the Mta and DBP promoters in cells genetically intact for RBP-Jk (Liang et al., 2002). K-Rta and RBP-Jk formed a complex with an RBP-Jk site in EMSA assays. Together with studies of the PAN promoter, these data suggested that K-Rta responsive promoters fall into two classes, differentiated by their dependence on RBP-Jk for specification (Liang et al., 2002).

\section{Rta STIMULATES DNA BINDING OF RBP-Jk}

While it was firmly established that K-Rta transactivation of the Mta promoter is RBP-Jk-dependent, mutations upstream of the RBP-Jk site in the Mta 5D promoter also reduced K-Rta transactivation, suggesting additional complexity in the K-Rta/RBPJk/DNA model for promoter specification (Lukac et al., 2001). Two complementary approaches revealed that the unexplained mutations affected Rta DNA binding (Carroll et al., 2006): (1) analyses of constitutive activators of the Notch pathway, and (2) comparison of DNA binding specificity of fully pure K-Rta and RBP-Jk.

The canonical model for Notch signal transduction holds that RBP-Jk is constitutively bound to DNA as a transcriptional repressor, but provides a nucleation site for activated Notch1 (NICD1) to activate transcription (Tanigaki and Honjo, 2010). The RBP-Jk site in the Mta promoter (Figure 2) is a perfect match to the consensus sequence (Liang et al., 2002). Therefore, it was surprising that both NICD1 and transcriptionally active RBP-Jk (WT RBP-Jk fused to the HSV VP16 transcriptional activation domain, called "RBP-Jk/VP16") failed to transactivate the native Mta promoter, nor the dimerized Mta 5D element (Carroll et al., 2006). As a positive control, both proteins activated the dimerized RBP-Jk element from the EBV Latency C promoter (Cp). These data suggested that RBP-Jk did not constitutively bind to the Mta promoter in vivo, an exception to the canonical model for Notch regulation.

Remarkably, co-expression of the K-Rta transcriptional mutant Rta $\triangle$ STAD, which contained K-Rta's domains for DNA binding, RBP-Jk interaction, and oligomerization (Figure 1), dramatically rescued the ability of RBP-Jk/VP16 to activate transcription of the native Mta promoter and the dimerized Mta 5D element (Carroll et al., 2006). These data suggested that Rta $\triangle$ STAD stimulated DNA binding of RBP-Jk/VP16 in vivo. Proving that Rta $\triangle$ STAD could similarly stimulate DNA binding of endogenous, WT RBP-Jk to DNA, Rta $\triangle$ STAD also rescued the ability of NICD1 to activate the native Mta promoter (Carroll et al., 2006; Palmeri et al., 2011). This effect of Rta $\triangle$ STAD was promoter specific: although the viral TK promoter also contains a consensus RBP-Jk site, and RBP-Jk/VP16 also failed to transactivate TK, Rta $\triangle$ STAD did not rescue RBPJk/VP16 transactivation of TK (Carroll et al., 2006). Rta $\Delta$ STAD also failed to stimulate transactivation of a Gal4 reporter by Gal4/VP16, proving that the effect of Rta $\triangle$ STAD on RBP-Jk/VP16 requires the RBP-Jk portion of the VP16 fusion molecule (Palmeri et al., 2011).

Clarification of the relationship between DNA binding specificity of RBP-Jk and K-Rta to the Mta 5D element came from studies of truncated K-Rta (aa 1-272; Figure 1) and RBP-Jk expressed and purified from E. coli (Carroll et al., 2006). As expected, RBPJk bound specifically to its consensus element, while Rta bound specifically to the A/T-rich sequences upstream of the RBP-Jk site in the $5 \mathrm{D}$ element. Mutational analyses of $5 \mathrm{D}$ showed (1) a strict correlation between RBP-Jk DNA binding and K-Rta transactivation, and (2) reduced, transactivation of $5 \mathrm{D}$ mutants that bound to RBP-Jk but not Rta. These data confirmed that RBP-Jk DNA binding was essential for K-Rta activation of the $5 \mathrm{D}$ element, and that DNA binding of Rta independently of RBP-Jk was not sufficient to activate the dimerized 5D element (Carroll et al., 2006). 
Combinatorial DNA binding of K-Rta and RBP-Jk revealed that ternary Rta/RBP-Jk/DNA complexes formed only on WT and mutant $5 \mathrm{D}$ elements to which both proteins bound independently (Carroll et al., 2006). Furthermore, Rta $\Delta$ STAD stimulation of RBP-Jk/VP16 binding also required intact binding sites for both proteins, and corresponded to maximal transactivation of these dimerized elements by WT K-Rta. C-terminal truncation of Rta $\triangle$ STAD to aa 414 generated a mutant of Rta that retained the ability to bind to the 5D element, but failed to form a ternary complex with RBP-Jk and DNA. This Rta mutant also failed to stimulate transactivation of 5D dimers by RBP-Jk/VP16 (Figure 2; Carroll et al., 2006). These data established that Rta $\triangle$ STAD stimulation of RBP-Jk/VP16 DNA binding required DNA/protein and protein/protein interactions of both proteins.

Proving that cognate, full-length K-Rta could also stimulate DNA binding of RBP-Jk, chromatin immunoprecipitations (ChIPs) showed that RBP-Jk is virtually undetectable on the Mta, $\mathrm{K}-\mathrm{bZIP}$, and K14 promoters during KSHV latency, but is enriched on these promoters during reactivation in a K-Rta-dependent fashion (Carroll et al., 2006). Rta also selectively stimulated RBP-Jk binding to the cellular IL- 6 and HES-1, but not CD23, promoters (Carroll et al., 2006). The inability of the RBP-Jk-dependent transactivators NICD1 and Esptein-Barr virus (EBV) nuclear antigen (EBNA)-2 to reactivate KSHV from latency when expressed ectopically in PEL cells (Liang and Ganem, 2003; Chang et al., 2005a; Carroll et al., 2006) confirmed that RBP-Jk is not bound to essential KSHV genes during latency in the absence of K-Rta.

\section{COMPARISON OF RBP-JK-DEPENDENT AND INDEPENDENT PROMOTERS}

K-Rta promoter binding and transactivation were compared for the PAN, kaposin, ORF 57, and vIL-6 genes. Nuclear run-on assays from KS-1 PEL cells that were treated with sodium butyrate demonstrated a hierarchy of transcription initiation rates for the genes, in the order PAN > kaposin > ORF57 > vIL-6 (Song et al., 2003). The hierarchy was similar to the magnitude of transactivation of the promoters of these genes in transient reporter assays in 293T, BJAB, and PEL cells. The hierarchy was also similar in comparative DNA binding affinities of K-Rta to short promoter elements from these genes. In particular, K-Rta's DNA binding affinity for PAN was at least 10-fold greater than for a 30-bp Mta element (similar to Mta 5D; Figure 2; Song et al., 2003). Notably, the differences in DNA binding affinities were greater than the differences in transactivation.

The Mta 5D element contained significant homology to the promoter of the KSHV macrophage inflammatory protein (vMIP)-I gene (Chang et al., 2005c). The homologous portion of the vMIP-I promoter conferred K-Rta responsiveness on a heterologous promoter. Rta aa 1-490/VP16, expressed in cultured mammalian cells, did not bind to $39 \mathrm{bp}$ K-Rta responsive elements from the Mta and vMIP-I promoters, although it bound to the PAN and kaposin elements (Chang et al., 2005c). A series of proteins from cellular extracts, including RBP-Jk, bound to the vMIP-I and Mta elements (Chang et al., 2005c). The K-Rta point mutation R160A abolished DNA binding of K-Rta aa 1-490/VP16, reduced transactivation of the short Mta and vMIP-I reporters, and eliminated transactivation of the short PAN and kaposin reporters
(Chang et al., 2005c). A series of additional mutations in K-Rta's DNA binding domain had inconsistent effects on DNA binding of Rta aa 1-490/VP16 and transactivation of the short Mta and vMIP-I reporters. Two of the Rta DNA binding mutants, R160A and R161A, showed reduced and delayed transactivation of the viral transcripts from the viral genome in PEL cells (Chang et al., 2005c). The transcription was probably dependent on WT Rta induced from the endogenous virus (see Conclusion and Perspectives). The authors concluded that DNA binding was essential for K-Rta to transactivate the PAN and kaposin elements, but not the Mta and vMIP-I elements (Chang et al., 2005c).

\section{K-Rta BINDS WITH HIGH AFFINITY TO CANT REPEATS IN THE Mta PROMOTER TO STIMULATE DNA BINDING OF RBP-Jk}

A puzzling observation was that K-Rta-stimulated RBP-Jk DNA binding to the Mta promoter (Carroll et al., 2006) yet bound with relatively low affinity to the Mta 5D DNA element (Song et al., 2003). Studies of the K-bZIP promoter provided a hint to resolve this conundrum. In the K-bZIP promoter, an extended repeat of phased A/T trinucleotides were bound by K-Rta with high affinity, but a single unit of that repeat bound to K-Rta with only low affinity (Figure 3; Liao et al., 2003a). The Mta promoter contains a similar A/T repeat (Liao et al., 2003a), and the 5D element contains only a single A/T unit (Figure 4A, bold sequence; Palmeri et al., 2011).

To determine the qualitative interactions of K-Rta with the Mta promoter, the DNAse I footprint of recombinant Rta $\triangle$ STAD was determined (Palmeri et al., 2011). WT Rta $\Delta$ STAD, but not a DNA binding mutant, footprinted four distinct stretches of the promoter (Figure $4 \mathrm{~A}$, underlined sequence). The foot print extended over $64 \mathrm{bp}$ flanking the RBP-Jk binding site, within the $-136 /-62$ portion of the promoter. The longest contiguous portion of the footprint protected the previously identified $5 \mathrm{D}$ element (Figure 4A). Alignment of the foot printed regions revealed a 14-nt consensus sequence, ANTGTAACANT ${ }^{\mathrm{A}} / \mathrm{T} / \mathrm{T} \mathrm{T}$, in which $N=$ any base (Figure 3; Palmeri et al., 2011). The consensus was repeated seven times in the foot printed region, and two pairs of two of the repeats formed palindromes (Figure 4A; Palmeri et al., 2011). Each unit contained the core sequence "CANT," so was termed the "CANT repeat." (Palmeri et al., 2011). Using saturating K-Rta concentrations, the footprint extended farther downstream to -21 to cover additional CANT repeats (Palmeri et al., 2011); this downstream region has been identified as an additional Rta responsive element (Wen et al., 2009).

\begin{tabular}{|rc|}
\hline Liao, et al., 2003a & $\begin{array}{r}{[\mathrm{A} / \mathrm{T}]_{3}-\mathrm{N}_{7}-[\mathrm{A} / \mathrm{T}]_{3}-\mathrm{N}_{7}-[\mathrm{A} / \mathrm{T}]_{3}} \\
{[\mathrm{~A} / \mathrm{T}]_{3}-\mathrm{N}_{17}-[\mathrm{A} / \mathrm{T}]_{3}}\end{array}$ \\
Chen, et al., 2009 & TTCCAGGAT $\mathrm{N}_{0-16}$ TTCCTGGGA \\
Ziegelbauer, et al., 2006 & CCCACTTT \\
Palmeri, et al., 2011 & ANTGTAACANT ${ }^{\mathrm{A}} / \mathrm{T}_{\mathrm{T}}^{\mathrm{A}}{ }_{\mathrm{T}} \mathrm{T}$ \\
& \\
FIGURE 3 | Comparison of consensus K-Rta binding sites. The indicated \\
K-Rta consensus sequences were derived from the listed studies. " $\mathrm{N}$ " \\
means any nucleotide.
\end{tabular}



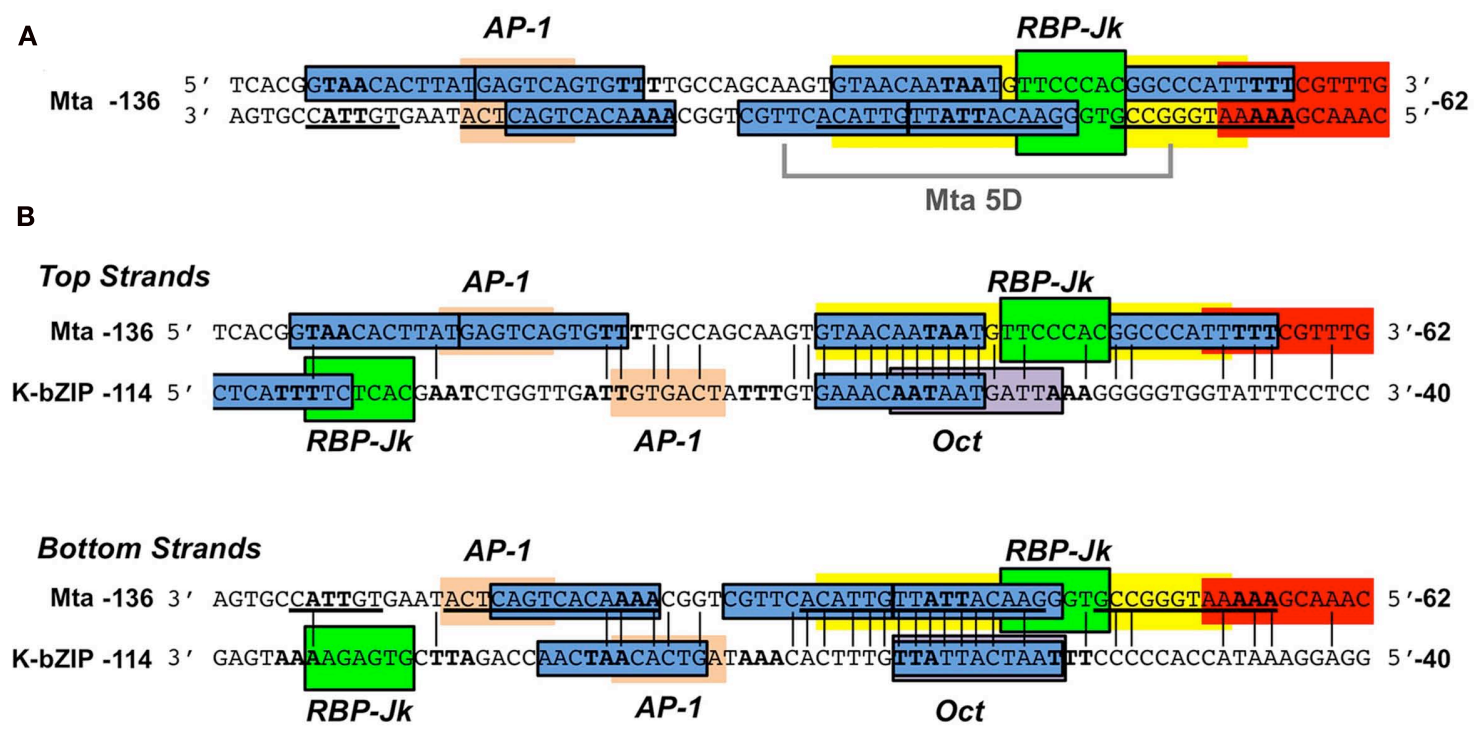

FIGURE 4 | Comparison of Mta and K-ZIP promoters. (A). Schematic of the $-136 /-62$ Mta promoter. Sequences of the top and bottom strands of the indicated portion of the Mta promoter. Numbers indicate positions relative to Mta transcriptional start site. Bold letters indicate four units of the $A / T_{3}$ trinucleotide repeat. Underlines indicate Rta's footprint from the bottom strand. Blue boxes indicate regions of highest conservation, nt 4-14, for each unit of the CANT repeat. The green box indicates the RBP-Jk binding site. The peach box indicates the AP-1 binding site. The yellow box indicates the K-RBP binding site. The red box indicates the IRF-7 binding site. The position of the short, Mta 5D element is indicated by the brackets. (B) Alignment of the top and bottom strands of the Mta and K-bZIP promoters. Numbering and box designations are as listed in the legend for Figure 3, above. The purple box indicates the Oct-1 binding site.
Rta $\triangle$ STAD's affinity for Mta $-136 /-62$ was $5.7 \mathrm{nM}$ (Palmeri et al., 2011), similar to K-Rta's affinity measured for its shorter PAN promoter binding site (Song et al., 2003). Rta $\Delta$ STAD's affinity for $-136 /-62$ was approximately 35 -fold greater than for the 5D element alone (Palmeri et al., 2011), similar to the affinity measured for a short Mta element in (Song et al., 2003). RBP-Jk enhanced Rta binding affinity fivefold to Mta $-136 /-62$, and ternary Rta/RBPJk/DNA complex formation on $-136 /-62$ was more robust than on the short 5D DNA (Palmeri et al., 2011). Therefore, classification of the Mta promoter as a high or low affinity K-Rta binding site depended on the length of DNA used as probe (Palmeri et al., 2011).

To test the functional significance of these data, a series of promoters were generated by artificially combining CANT repeats and RBP-Jk sites, or mutating the cognate Mta promoter to alter the number and architecture of CANT repeats (Palmeri et al., 2011). Each was tested for transactivation by the combination of Rta $\triangle$ STAD and RBP-Jk/VP16. The data confirmed that K-Rta's stimulation of RBP-Jk DNA binding required an intact RBPJk binding site. However, K-Rta's stimulation of RBP-Jk DNA binding was proportional to the number and position of CANT elements for K-Rta, but not RBP-Jk sites. Furthermore, the number of CANT repeats, their presence in palindromes, and their positions relative to the RBP-Jk binding site determined the optimal target for Rta stimulation of RBP-Jk DNA binding. Both CA dinucleotides and A/T-richness of the promoter were critical for the Rta/RBP-Jk/DNA interaction (Palmeri et al., 2011).

The minimum promoter structure required for robust Rta/RBP-Jk transactivation contained a single RBP-Jk site straddled by two pairs of palindromic CANT repeats (Palmeri et al., 2011). DNA binding and tetramerization mutants of KRta failed to stimulate RBP-Jk DNA binding (Palmeri et al., 2011). Based on these genetic studies of K-Rta, RBP-Jk, and the Mta promoter, a model was proposed in which K-Rta tetramers make high affinity interactions with repetitive DNA elements in KSHV promoters (Figure 5; Palmeri et al., 2011). Tetramerization of Rta allows it to straddle RBP-Jk and contact palindromic pairs of CANT repeats flanking RBP-Jk in the Mta promoter. Two regions of RBP-Jk independently bind to K-Rta in solution (Liang et al., 2002; Carroll et al., 2006) permitting Rta bound to DNA on both sides of RBP-Jk to contact RBP-Jk simultaneously. A second K-Rta tetramer might bind to the upstream CANT repeats, with or without an unidentified cellular protein, to further stabilize K-Rta DNA binding and recruitment of RBP-Jk to DNA (Figure 5). Since the upstream CANT repeats partially overlap a functional binding site for the cellular protein AP-1, and AP-1 enhances K-Rta transactivation (Wang et al., 2004a), AP-1 might contribute to formation of the Rta/RBP-Jk/DNA complex.

Chromatin immunoprecipitations showed that RBP-Jk DNA binding was broadly, but selectively, stimulated across the entire KSHV genome during reactivation (Palmeri et al., 2011). Bioinformatics showed that CANT and A/T repeats were located in proximity to many RBP-Jk sites throughout the KSHV genome. This study thus integrated high affinity Rta DNA binding with the requirement for RBP-Jk in Rta transactivation, to define the promoter requirements for formation of transcriptionally productive Rta/RBP-Jk/DNA complexes (Palmeri et al., 2011). 


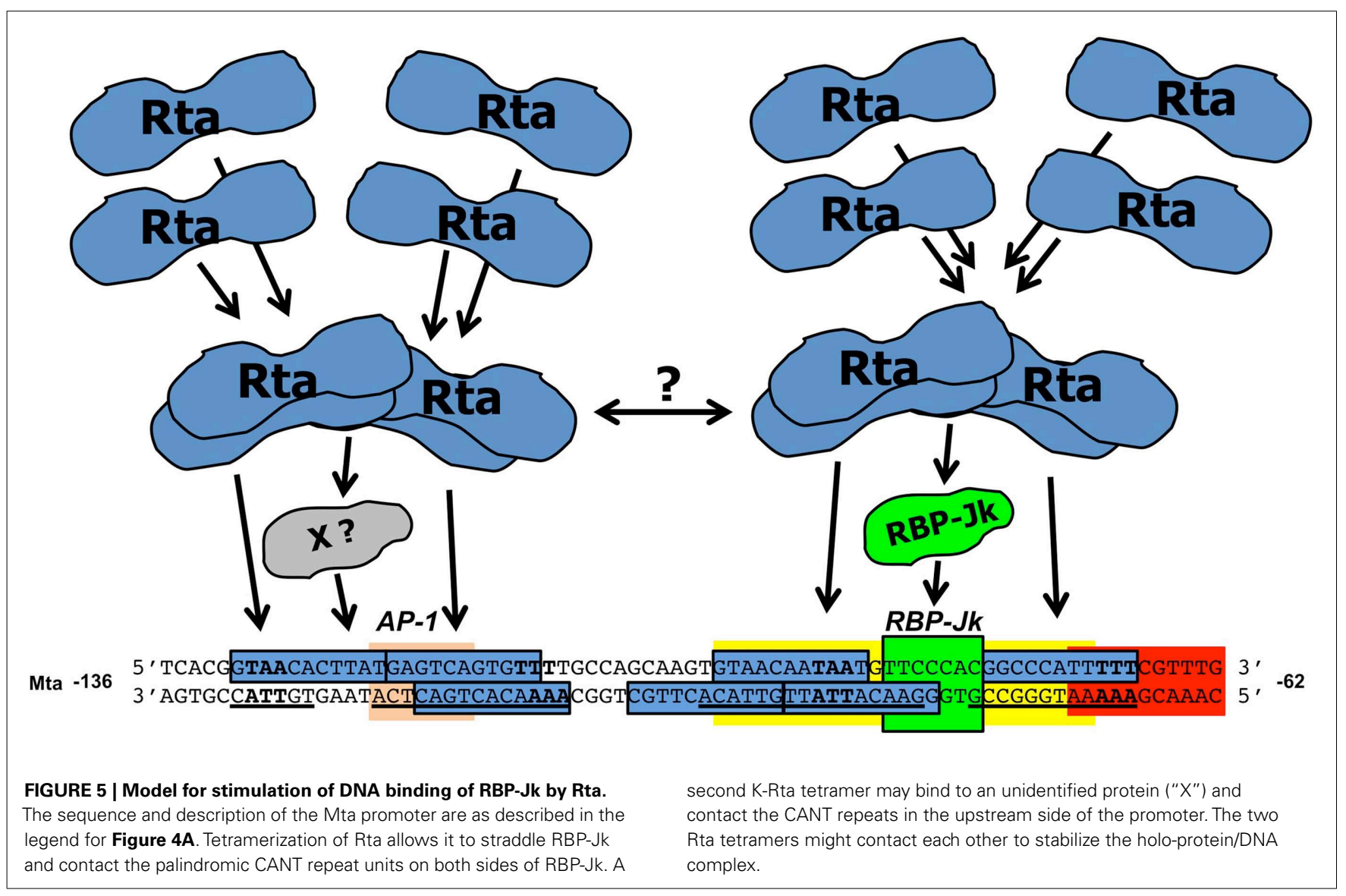

The PAN and Mta promoter models for Rta DNA binding therefore suggest dramatic differences in mechanisms for forming productive Rta/DNA transcription complexes. The PAN promoter contains a short, high affinity K-Rta binding site from which Rta activates transcription independently of RBP-Jk (Liang et al., 2002; Song et al., 2003). The Mta promoter contains multiple Rta CANT binding sites, each of which is an independent, low affinity binding site, but which act in concert as a high avidity, extended element that bind K-Rta multimers simultaneously, and together with RBP-Jk (Figure 5; Palmeri et al., 2011).

\section{RBP-Jk AND OCTAMER-1 PARTICIPATE IN K-Rta SPECIFICATION OF THE K-bZIP PROMOTER}

Although CANT repeats are found throughout the KSHV genome (Palmeri et al., 2011), their significance for Rta transactivation has only been formally investigated for the Mta and K-bZIP promoters (see Mta, above). The core sequences of two of the Mta CANT repeats are homologous to the TATA proximal K-bZIP promoter (Figure 4B; Lukac et al., 2001). These K-bZIP repeats also bound to recombinant K-Rta purified from E. coli. Mutation of the repeats in the full-length K-bZIP promoter reduced K-Rta-mediated transactivation by greater than $80 \%$ (Lukac et al., 2001; Carroll et al., 2007).

In the K-bZIP promoter, the CANT repeats overlap an RBP-Jk binding site and a sequence that is well conserved with binding sites for the cellular Octamer (Oct) transcription factors that are found in the immediate early promoters of herpes simplex virus type 1 (HSV-1; Figure 4B; Carroll et al., 2007). At least seven protein complexes from infected PEL cells bound to a 26-bp DNA from the K-bZIP CANT repeats (Carroll et al., 2007). Mutation of the K-bZIP sequence eliminated binding of one cellular complex. That complex was super shifted by an Oct- 1 specific antibody and was increased following TPA addition to PEL cells. Together, these data suggested that Oct- 1 binds to the K-bZIP promoter in a manner that corresponds to viral reactivation and Rta transactivation (Carroll et al., 2007).

Recombinant K-Rta and Oct-1 bound to the WT but not mutant promoter, and Oct-1 and Rta were specifically ChIP'd on the K-bZIP promoter in reactivating cells (Carroll et al., 2007). Their binding sites overlap (Figure 4B). Recombinant Oct-1 enhanced Rta DNA binding to the promoter, and co-expression of Oct-1 enhanced Rta-mediated transactivation of the wild type but not the mutant K-bZIP promoter.

Octamer-1 and WT Rta proteins bound to each other directly in vitro (Carroll et al., 2007). aa 134-150 of Rta, within its DNA binding domain, contains a sub-domain homologous to the Octinteracting domains (OIDs) of the Yeast protein MAT $\alpha 2$ and the HSV-1 virion protein 16 (Figure 1). A series of single point mutations of Rta's OID had individual, negative effects on either DNA or Oct-1 binding, but none completely eliminated transactivation of K-bZIP and viral reactivation (Carroll et al., 2007). However, combining 3 of these point mutations, or insertion of a 4 aa peptide, ablated Rta DNA and Oct- 1 binding, transactivation of the K-bZIP promoter and viral reactivation. None of the 
mutations inhibited Rta's interaction with RBP-Jk (Carroll et al., 2007).

A second Oct-1 binding site was identified upstream of the CANT repeats (Carroll et al., 2007). In transfections of B cells, mutation of both Oct sites ablated Rta-mediated transactivation (Carroll et al., 2007). However, in transfections of WT and mutant MEFs, Oct-1 deletion only reduced Rta transactivation of $\mathrm{K}$ bZIP by $50 \%$, while RBP-Jk deletion eliminated transactivation. These data suggested that the Rta/Oct-1 interaction is essential for optimal KSHV reactivation, and the requirement for Oct-1 in Rtamediated transactivation was stricter in B cells than MEFs (Carroll et al., 2007). Oct-1 is also involved in K-Rta transactivation of the K-Rta and K9 promoters (Sakakibara et al., 2001; Ueda et al., 2002; Harrison and Whitehouse, 2008).

\section{K-Rta DNA BINDING SCREENS}

Two genome-wide screens were performed using distinct strategies to identify K-Rta DNA binding sequences. In one strategy, FLAG-tagged K-Rta aa 1-390 was stably expressed in PEL cells, and chromatin was ChIP'd with anti-FLAG antibody $6 \mathrm{~h}$ after adding TPA to the cells (Chen et al., 2009). ChIP'd sequences were detected by a KSHV genomic tiling array. Nineteen viral loci scored above background, and were compared to previously published Rta binding sites to derive a consensus. The consensus contained two imperfect inverted tandem repeats with the sequence TTCCAGGAT $(\mathrm{N})_{0-16}$ TTCCTGGGA (Figure 3). The study identified a new Rta responsive element within the first intron of the K15 P gene.

In the other strategy, Rta $\triangle \mathrm{STAD}$ generated in E. coli was incubated with sheared KSHV genomic DNA, and bound DNA was enriched by serial repetition of PCR amplification and incubation with Rta $\triangle$ STAD (Ziegelbauer et al., 2006). Bound sequences were detected using a KSHV genomic microarray. Eighteen viral loci scored above background. The highest scoring sequence was in the gB promoter, which the authors confirmed was strongly activated by K-Rta. The authors aligned gB with other known transcriptional targets of Rta and identified a consensus with the sequence $\mathrm{C} / \mathrm{T} \mathrm{C}^{\mathrm{A} / \mathrm{C}} \mathrm{C} / \mathrm{T}$ TTT (Figure 3 ). Notably, the core of this consensus is a CANT element (Figure 3).

\section{OTHER MECHANISMS FOR K-Rta PROMOTER SPECIFICATION}

WT K-Rta, but not a DNA binding mutant, induces the expression of IFN-stimulated genes (Zhang et al., 2005). K-Rta's binding sites are similar to interferon stimulated response elements (ISREs), and K-Rta activates the K14-ORF 74 promoter through an ISRElike element. K-Rta's DNA binding domain is partly homologous to that of cellular interferon regulatory factor (IRF) proteins, and mutations in conserved aas inhibit K-Rta DNA binding (Zhang et al., 2005). K-Rta also binds to DNAs containing sites matching the A/T trinucleotide consensus (Liao et al., 2003a; Figure 3) to activate transcription of the KSHV K1 promoter (Bowser et al., 2006).

K-Rta interacts with the cellular protein $\mathrm{C} / \mathrm{EBP} \alpha$ to regulate transactivation of various KSHV promoters (Wang et al., 2003a,b). In transient transfections, $\mathrm{C} / \mathrm{EBP} \alpha$ and Rta cooperate to activate transcription of the K-bZIP, Mta, Rta, and PAN promoters. All of these promoters contain at least one $\mathrm{C} / \mathrm{EBP} \alpha$ binding site required for contributing to Rta-mediated transactivation. However, since the consensus binding site for $\mathrm{C} / \mathrm{EBP} \alpha$ (the CAAT box Osada et al., 1996) matches the core of the K-Rta binding CANT repeats (Palmeri et al., 2011; Figure 3), CAAT box mutations also have the potential to disrupt binding of K-Rta. K-Rta binds directly to $\mathrm{C} / \mathrm{EBP} \alpha$ in vitro and in vivo, and deletion of aa 151-167 of K-Rta inhibits (1) the interaction of K-Rta with $\mathrm{C} / \mathrm{EBP} \alpha$ or PAN DNA (Figures 1), (2) transactivation of the PAN promoter by Rta alone, and (3) cooperative transactivation of the K-bZIP or PAN promoters by Rta with $\mathrm{C} / \mathrm{EBP} \alpha$ (Wang et al., 2003a,b) ChIPs demonstrated that $\mathrm{C} / \mathrm{EBP} \alpha$ and Rta bound to the Rta, PAN, and Mta promoters in increasing amounts at 8 and $24 \mathrm{~h}$ after TPA addition to PEL cells, but not in un-treated cells (Wang et al., 2003b). Immunodepletion of $\mathrm{C} / \mathrm{EBP} \alpha$ prior to Rta ChIP suggested that it participated in binding of Rta to the K-bZIP promoter. K-Rta may also bind to the ori-Lyts through $\mathrm{C} / \mathrm{EBP} \alpha$ sites (Wang et al., 2006; Kato-Noah et al., 2007).

K-Rta also cooperates with AP-1 constituents cJun and cFos to activate transcription (Wang et al., 2004a). Rta binds to cJun and cFos in vivo and in vitro, and the three proteins cooperatively transactivate the K-bZIP, Mta, and Rta promoters in uninfected cells. cJun ChIPs those promoters in TPA-treated, but not un-treated, BCBL-1 cells.

K-Rta also transactivates STAT-driven reporter genes by stimulating STAT3 dimerization and nuclear localization (Gwack et al. 2002; Figure 1).

\section{K-Rta HAS THE POTENTIAL TO ACTIVATE KSHV GENE EXPRESSION BROADLY DURING INFECTION}

Transcriptomic analyses indicated that expressing Rta ectopically in PEL cells induced a coordinated cascade of KSHV gene expression (Nakamura et al., 2003; Damania et al., 2004). Transient expression of Rta induced all ORFs except the latency genes (Damania et al., 2004), while uniform expression of K-Rta under the control of doxycycline (dox) induced every viral ORF within $48 \mathrm{~h}$ after dox addition (Nakamura et al., 2003). In the latter study, the productive transcriptional cascade induced by dox was more rapid and robust than that induced by TPA treatment, suggesting TPA is a relatively inefficient reactivation signal. Most of the viral homologs of cell growth regulatory genes were expressed as DE transcripts, and infectious viral progeny were produced.

K-Rta activates many KSHV promoters in an RBP-Jkdependent manner (Liang et al., 2002; Liang and Ganem, 2004; Chang et al., 2005b, 2010; Lan et al., 2005a; Carroll et al., 2006, 2007; Wang and Yuan, 2006; Ziegelbauer et al., 2006; Liu et al., 2008; Persson and Wilson, 2010; Wang et al., 2010). At least 260 putative binding sites for K-Rta's cellular binding partner RBP-Jk were identified in silico in the KSHV genome (Persson and Wilson, 2010 and Gonzalez-Lopez and Lukac, unpublished). Three genomic regions with clustered sites were identified as new RBP-Jk-dependent transactivation targets of K-Rta (Persson and Wilson, 2010). Among the putative RBP-Jk sites, the RBP-Jk protein demonstrated a binding preference, and K-Rta demonstrated a transactivation preference. A signature was derived for 17 confirmed RBP-Jk binding sites, which added a cytosine residue at position upstream of the canonical GTGGGAA core sequence (Persson and Wilson, 2010). Further work is needed to determine which of the putative 
RBP-Jk sites are co-regulated by K-Rta DNA binding. The observation that the RBP-Jk sites are scattered widely across the unique portion of the genome suggests that K-Rta has the potential to activate transcription of every KSHV ORF during reactivation.

A comprehensive strategy to identify all of K-Rta's transcriptional targets compared 83 putative KSHV promoters for K-Rta transactivation in uninfected 293 cells, with ChIP of dox-inducible Rta from infected PEL cells (Ellison et al., 2009). Rta transactivated 34 of the promoters greater than 10 -fold, including many previously identified targets of K-Rta. At $12 \mathrm{~h}$ following dox induction, K-Rta was significantly ChIP'd on 22 promoters (Ellison et al., 2009). Fourteen of the promoters corresponded to those identified as targets in 293 cells. Although there was little correspondence between magnitude of transactivation and magnitude of ChIP enrichment, there were cell-specific differences in specificity and magnitude of transactivation (Ellison et al., 2009). A subset of promoters were tested at later time points following K-Rta induction, which indicated that K-Rta associated with increasingly greater numbers of promoters as the infection progressed (Ellison et al., 2009). These data suggest that K-Rta has the potential to activate at least half of putative KSHV promoters during reactivation.

\section{DIRECT K-Rta TRANSACTIVATION IS LIMITED IN INFECTED CELLS}

It is well established that K-Rta is the only viral protein necessary and sufficient to initiate the complete KSHV productive cycle. The foundation of this process is the ordered expression of viral genes, with transcriptional initiation of specific genes one of the earliest regulated steps. It was clear that the gene-specific transcriptional cascade could be classified kinetically from the earliest detection of Rta to the replication-dependent expression of the true late genes. It was also clear that Rta had the potential to activate transcription of many, if not most, of the viral genes. However, it was not clear which viral genes Rta directly transactivated during reactivation in infected cells, and which depended on prior expression of Rta's targets.

To answer this question, a modified allele of Rta was engineered that was fully functional, but could be conditionally activated in the presence of protein synthesis inhibitors to eliminate any other protein expression (Bu et al., 2008). The strategy relied upon manipulation of Rta's nuclear/cytoplasmic localization by fusion to the hormone-binding domain of the murine estrogen receptor gene (ER), an approach used successfully for many other transcription factors. In this approach, nuclear transcription factors are constitutively localized to the cytoplasm by fusion to the ER, but re-localize to the nucleus in the presence of 4-hydroxytamoxifen (OHT).

However, fusion of the WT K-Rta protein to ER failed to localize the protein to the cytoplasm, suggesting that Rta's cognate NLS was dominant (Bu et al., 2008). Sequence analysis identified two putative nuclear localization signals in K-Rta, between aa 6-12 (NLS1) and 516-530 (NLS2; Figure 1). NLS1 regulated nuclear localization in one publication (Chen et al., 2000), but deletion of NLS1 had no effect on Rta-mediated function in CV-1, BL-41, and PEL cells (Bu et al., 2008). Instead, deletion of aa 516-530 (NLS2) eliminated both K-Rta-mediated transactivation and reactivation (Bu et al., 2008). Deletion of NLS2 re-localized full-length K-Rta to the cytoplasm, and fusion of NLS2 to GFP re-localized GFP to the nucleus. NLS2 was thus a bona fide nuclear localization signal for K-Rta (Bu et al., 2008; Figure 1). WT K-Rta and each NLS mutant were tested as independent fusions to ER to determine sub-cellular localization and transactivation of Mta and PAN reporter plasmids. In this experiment, K-Rta with both putative NLSs deleted (Rta $\triangle$ NLS1,2-ER) was fully regulatable by addition or subtraction of OHT to growth media of transfected cells (Bu et al., 2008).

The effect of the regulatable K-Rta allele on KSHV was tested using Vero cells infected with the recombinant virus rKSHV.219. This virus constitutively expresses GFP in all infected cells, and conditionally expresses RFP from a second copy of the PAN promoter as a reporter for viral reactivation (Vieira and O'Hearn, 2004). The Rta $\Delta$ NLS1,2-ER fusion protein demonstrated OHTdependent stimulation of the complete viral reactivation cycle, as determined by expression of viral RNAs and proteins, RFP induction, and production of infectious virus in the cell supernatant (Bu et al., 2008).

To determine which viral genes were transactivated by Rta in the absence of any other protein expression, Rta $\Delta$ NLS1,2-ER was expressed in Vero-rKSHV.219 cells for $3 \mathrm{~h}$ prior to addition of the protein synthesis inhibitor hygromycin to the cells (Bu et al., 2008). Shut-off of protein synthesis was confirmed by lack of endogenous RFP and tubulin expression, and lack of luciferase and beta-galactosidase expression from ectopic reporter plasmids. Fifteen hours following hygromycin addition, OHT was added to half of the cultures. Total RNA was harvested from cells, and analyzed using a printed oligonucleotide microarray containing detectors for the entire KSHV transcriptome. Only eight genes were significantly induced by nuclear re-localized K-Rta in the absence of de novo protein synthesis: PAN, Mta, ORF 56, vIL-6, ORF 37, K14, K9, and ORF 52 (Bu et al., 2008).

This small number of direct K-Rta targets in infected cells is remarkable considering that the KSHV genome contains at least 260 putative RBP-Jk binding sites, and Rta activates or binds to approximately half of the KSHV promoters in transiently transfected, uninfected cells. These data suggest that additional viral and cellular protein expression is required for the lytic cycle to progress beyond these eight direct transcriptional targets of Rta. The data also support a model in which KSHV reactivation is controlled not only by induction of K-Rta transcription, but also by regulation of K-Rta protein function in a manner that requires on-going translation. In this scenario, K-Rta expression is not sufficient to ensure completion of the entire lytic cascade, but K-Rta's function is regulated to direct the progression of reactivation.

\section{REGULATORS OF K-Rta FUNCTION MTA COOPERATES WITH Rta AS A COMMITMENT FACTOR FOR REACTIVATION}

KSHV Mta was identified as one of eight direct transcriptional targets of K-Rta during KSHV reactivation (Bu et al., 2008). Mta's promoter has served as a model for understanding K-Rta's specification of transactivation (see "RBP-Jk-dependent promoters," above; Figure 4A). Genetic studies demonstrated that Mta is required for productive viral reactivation, especially for optimal expression of DNA replication factors (Han and Swaminathan, 2006; Majerciak et al., 2007). Mta was unable to reactivate KSHV 
from latency when ectopically expressed in PEL cells, but synergized with K-Rta to stimulate reactivation (Lukac et al., 1998; Palmeri et al., 2007). Synergy with K-Rta was detected by expression of the PAN RNA from the endogenous viral genome in PEL cells, and release of mature virus to the cell medium (Palmeri et al., 2007).

Mta is encoded by KSHV ORF 57, and is homologous to regulators of gene expression in other herpesviruses. In KSHV, ORF 57 is one of the first genes transcribed during reactivation, following transcription of K-Rta and other IE genes (Lukac et al., 1999). The Mta protein localizes to the nuclei of cells transfected with an ORF 57 expression vector (Kirshner et al., 2000; Majerciak et al., 2006b). The major monocistronic transcript that encodes ORF 57 is $1.4 \mathrm{~kb}$ and contains a single, small $5^{\prime}$ intron (Kirshner et al., 2000). Mta is expressed as a 49-kDa protein within $1 \mathrm{~h}$ of TPA treatment of PEL cells, following K-Rta protein expression (Palmeri et al., 2007). Up until $24 \mathrm{~h}$ post-TPA, total K-Rta and Mta proteins increased in abundance, and the percentages of cells expressing either Mta or Rta also increased (Palmeri et al., 2007). From 24 to $72 \mathrm{~h}$ post-TPA treatment, the percentage of KRta expressing cells continued to increase linearly to $20 \%$, while the percentage of Mta-expressing cells plateaued at 10-12\%. Mta expression thus marked a population of cells that diverged from $\mathrm{K}$-Rta positive cells as reactivation progressed (Palmeri et al., 2007).

To determine the relationship between K-Rta and Mta expression, and complete viral reactivation, the percentages of cells co-expressing either K-Rta or Mta, together with the true late protein K8.1, were measured (Palmeri et al., 2007). At all times post-TPA addition, the proportion of Mta positive cells that also expressed K8.1 exceeded that of K-Rta positive cells that expressed K8.1. Beginning at $24 \mathrm{~h}$ post-TPA, the proportion of Mta positive cells that also expressed K8.1 increased and continued for the duration of the experiment, while the percent of K-Rta positive cells that expressed K8.1 decreased during that time. At $72 \mathrm{~h}$ post-TPA, the latest time point tested, $80 \%$ of Mta positive cells also expressed K8.1, while only $20 \%$ of K-Rta positive cells expressed K8.1 These data showed that although K-Rta was necessary and sufficient to initiate KSHV reactivation, expression of Mta predicted successful progression of the productive cycle to the late stage (Palmeri et al., 2007). The expression of Mta therefore seemed to be required to commit a reactivating virus to the entire productive cascade.

KSHV Mta has multiple, pleiotropic effects on gene expression. Mta increased the steady-state abundances of the transcripts for ORF 59/58 and PAN, but not ORF 74/vGPCR or K5, when the transcripts were expressed from the CMV promoter in CV-1 cells (Kirshner et al., 2000). However, Mta showed inconsistent and $<2$-fold effects on firefly luciferase expressed under control of the kaposin, DNA polymerase, PAN, and TK promoters, in CV-1 cells (Kirshner et al., 2000). These data suggested a post-transcriptional role for Mta in transactivating gene expression.

Despite Mta's inability to independently transactivate $\mathrm{KSHV}$ promoters, Mta dramatically synergized with K-Rta in a promoter specific fashion (Kirshner et al., 2000; Palmeri et al., 2007). These effects of expressing K-Rta and Mta alone and together were similar to their effects on KSHV reactivation. Mta strongly synergized with K-Rta transactivation of the PAN promoter, and cooperated to a lesser amount for the ori-Lyt (L), Kaposin, ORF 57, and TK promoters (Kirshner et al., 2000; Palmeri et al., 2007). The effect on the PAN promoter was 80 - to 243 -fold over K-Rta transactivation alone. However, K-Rta/Mta did not synergize on the K-bZIP and ORF 50 promoters (Kirshner et al., 2000; Palmeri et al., 2007). K-Rta/Mta synergy required K-Rta transactivation, since synergy was eliminated by (1) mutation of the K-Rta binding site in the PAN promoter, and (2) co-expression of the K-Rta dominant negative mutant Rta $\Delta$ STAD (Kirshner et al., 2000). K-Rta/Mta synergy was reduced but not eliminated when an intron was added to the luciferase transcript in the PAN reporter plasmid (Kirshner et al., 2000). Mta did not increase the amount of K-Rta mRNA and protein levels in co-transfected cells (Kirshner et al., 2000). These data suggested that Mta has a K-Rta-dependent, promoter specific effect on transactivation in CV-1 cells, and the promoter specific effect of Mta is further influenced by the structure of the resulting transcript.

Mta binds directly to K-Rta, and requires two regions of Rta: the LR and aa 275-484 (Palmeri et al., 2007; Figure 1). K-Rta DNA binding was necessary for synergy with Mta, but it was not sufficient (Palmeri et al., 2007). Although K-Rta strongly transactivated the PAN promoter when truncated immediately upstream of the K-Rta binding site, or when the K-Rta binding site was cloned upstream of a heterologous TATA box, K-Rta/Mta synergy was severely reduced (Palmeri et al., 2007). Therefore, the K-Rta binding site, plus additional elements, in the PAN promoter were necessary for robust K-Rta/Mta synergy.

Further investigation of K-Rta/Mta synergy revealed a cellspecific, Rta-independent transcriptional activation function for Mta (Palmeri et al., 2007). Mta strongly activated the PAN promoter reporter in 293 cells and in Akata-31 cells, a sub-clone of Akata cells that were cured of EBV infection. Mta transactivation was promoter specific in 293 cells, and the WT and mutant promoters that were activated were those on which Mta synergized with Rta in CV-1 cells (Palmeri et al., 2007). Since Mta and KRta did not synergize in 293 cells, and Mta transactivation was independent of basal promoter activity, the data suggested that 293 cells endogenously mimic a K-Rta function required for Mta synergy in CV-1 cells.

Multiple experiments support a direct transcriptional role for Mta in transactivation. Mta transactivated the PAN promoter in nuclear run-on transcription assays, demonstrating that it is a bona fide transcriptional transactivator (Palmeri et al., 2007). Mta also activated the ORF 50 promoter in cooperation with Rta (Malik et al., 2004a). Mta and K-Rta ChIP'd the K-Rta and PAN promoters in KSHV infected cells (Malik et al., 2004a; Palmeri et al., 2007).

However, two observations supported an additional posttranscriptional role for Mta in synergy with K-Rta. The magnitude of Mta transactivation in the nuclear run-on experiments was considerably lower than in whole cell reporter assays, and Mta transactivation in the presence of actinomycin D suggested that Mta also stabilized reporter messages (Palmeri et al., 2007). Indeed, the Mta ORF includes protein domains with putative transcriptional and post-transcriptional functions, and Mta binds to proteins that regulate gene expression at various points (Malik et al., 2004a,b; 
Nishimura et al., 2004; Majerciak et al., 2006b, 2008, 2011; Boyne et al., 2010a; Jackson et al., 2011).

In fact, an expanding literature demonstrates multiple posttranscriptional roles for Mta. Mta enhances the accumulation of both viral (Kirshner et al., 2000; Han and Swaminathan, 2006; Majerciak et al., 2006a,c, 2008) and cellular (Gupta et al., 2000) RNAs in a gene-specific manner. Mta binds directly to RNAs in a sequence specific fashion to stabilize them or to promote escape from RNA silencing (Majerciak et al., 2006b; Nekorchuk et al., 2007; Sahin et al., 2010; Kang et al., 2011b; Massimelli et al., 2011; Sei and Conrad, 2011), enhances RNA splicing (Majerciak et al., 2008), shuttles between nuclei in heterokaryon assays (Bello et al., 1999), and promotes the nuclear export of un-spliced RNAs (Malik et al., 2004b; Boyne et al., 2008; Jackson et al., 2011; Majerciak et al., 2011; Taylor et al., 2011). Mta also stimulates mRNA translation (Nishimura et al., 2004; Boyne et al., 2010a). Details of Mta's posttranscriptional functions can be found in a number of excellent literature reviews (Swaminathan, 2005; Conrad, 2009; Majerciak and Zheng, 2009; Boyne et al., 2010b).

A model that is supported by all of the publications is that Mta is present at viral promoters prior to or during transcriptional initiation, the earliest step in biogenesis of an RNA. Mta might subsequently associate with the nascent RNA transcript in the nucleus, then remain associated with the transcript to regulate it post-transcriptionally. In this model, sequence specific transactivation by Mta could be governed by direct interaction with DNA or RNA, or indirectly by interaction with K-Rta.

\section{K-Rta AUTOREGULATES ITS FUNCTION}

K-Rta encodes two independent regions that auto-regulate its DNA binding and abundance (Chang and Miller, 2004; Chang et al., 2008). K-Rta aa 520-535 inhibits DNA binding to the PAN promoter in cis, and aa 590-650 destabilizes K-Rta in concert with a domain that overlaps aa 520-535 (Figure 1). K-Rta mutants in aa 520-535 are better DNA binders, but worse transactivators, of PAN, Mta, and vMIP-I. Fusion of the destabilizing element to glutathione-S-transferase (GST) destabilizes that protein, also. Since K-Rta's NLS2 is located at aa 516-530 (Figure 1), these functions of K-Rta might be co-regulated with proper sub-cellular localization. Further work is required to determine whether aa 590-650 are involved in K-Rta auto-ubiquitylation.

\section{NEGATIVE REGULATORS OF Rta FUNCTION}

Post-translational modifications and heterologous proteins inhibit K-Rta. The cellular protein IRF-7 competes with K-Rta for binding the Mta promoter to inhibit Rta-mediated transactivation (Wang et al., 2005). The IRF-7 binding site overlaps a CANT repeat downstream of the RBP-Jk site in the Mta promoter (Figure 4A).

The cellular NF-kB (RelA) protein inhibits K-Rta transactivation in 293 cells and reactivation in PELs, promoter binding by K-Rta and RBP-Jk, and K-Rta binding to RBPJk (Izumiya et al., 2009). RelA repression was promoter specific; it did not affect K-Rta transactivation of the Ori-RNA or Kaposin promoters. RelA bound directly to RBP-Jk. Multiple NF-kB family proteins inhibited Rta transactivation in 293 cells (Brown et al., 2003; Izumiya et al., 2009). The NF-kB inhibitor Bay11-7082 induced productive cycle gene expression in latently infected PEL cells, suggesting that high NF-kB levels help maintain latency (Brown et al., 2003). RelA also inhibited K-Rta transactivation of the human IL-6 promoter (Roan et al., 2002).

The cellular protein Trasducin-like enhancer of split (TLE) 2 binds directly to K-Rta aa 170-246 (Figure 1), and competes with K-Rta binding to RBP-Jk (He et al., 2010). TLE2 downregulates K-Rta transactivation of the K-Rta, PAN, K-bZIP, Mta, and ORF 59 promoters, and reduces K-Rta-mediated reactivation. Knockdown of TLE2 enhances KSHV reactivation. K-Rta/TLE2 complexes bind to the PAN, K-bZIP, and ORF 59 promoters, and TLE2 antibody ChIPs the PAN and Mta promoters during reactivation.

K-Rta's promoter association and stimulation of reactivation in PEL cells can be decreased by poly-ADP-ribosylation and phosphorylation of its serine-threonine (ST)-rich domain, mediated by binding to the cellular proteins poly(ADP-ribose) polymerase(PARP)-1 and human kinase from chicken (KFC; Gwack et al., 2003; Figure 1).

\section{DUAL POSITIVE/NEGATIVE REGULATORS OF K-Rta FUNCTION}

K-Rta makes complex interactions with the KSHV IE protein K-bZIP that are both positive and negative. In the screen of 83 putative KSHV promoters in 293 cells, K-bZIP activated 21 alone, synergized with Rta on 19, and repressed Rta transactivation of 3 (Ellison et al., 2009). K-bZIP's ability to repress K-Rta-mediated activation required a direct protein-protein interaction (Figure 1), and was not relieved by overexpressing transcriptional coactivators (Liao et al., 2003b). Three K-Rta deletions inhibited binding to K-bZIP: aa 1-109, 110-295, and 293-503.

The cellular protein KSHV Rta binding protein (K-RBP) binds K-Rta aa 1-272, and contains a Kruppel-associated box (KRAB) and 12 adjacent zinc finger motifs (Wang et al., 2001b; Figure 1). When expressed at low levels, K-RBP synergizes with K-Rta transactivation of the Mta, K-bZIP, vIL-6, K-Rta, and vMIP-1 promoters, but at high levels, represses K-Rta transactivation of K-bZIP, Mta, and PAN promoters (Wang et al., 2001b; Yang and Wood, 2007). K-RBP binds to DNAs that contain a GC-rich core, including a 40-bp region of the Mta promoter that contains the RBP-Jk site and overlaps CANT repeats. K-RBP competes with K-Rta to bind the Mta promoter, and represses KSHV lytic reactivation (Yang and Wood, 2007; Yang et al., 2009).

\section{Rta AND CHROMATIN}

Latency and reactivation of KSHV is associated with dynamic chromatin remodeling (Gunther and Grundhoff, 2010; Toth et al., 2010; Chang et al., 2011; Kang et al., 2011a). Early evidence for the dependence of reactivation on relief of repressive chromatin came from the observation that histone deacetylase inhibitors (HDACi) reactivate KSHV from latency (Sun et al., 1996). Dominant negative Rta $\triangle$ STAD inhibited reactivation stimulated by the HDACi sodium butyrate, suggesting that repressive chromatin controls expression of K-Rta from the latent genome (Lukac et al., 1999).

Similar to many DBPs, chromatin structure also plays an integral role in K-Rta's accessibility to DNA. ChIPs specific for histone $\mathrm{H} 3-\mathrm{K} 9$ acetylation (H3acK9), a marker for active chromatin, showed that at $12 \mathrm{~h}$ post-K-Rta induction, H3acK9 was ChIP'd 
on 48 promoters (Ellison et al., 2009). There was a correlation between the increase in active chromatin and progression of reactivation, and H3acK9 was ChIP'd before, or concurrent with, ChIP of K-Rta. The data suggested that chromatin accessibility regulated K-Rta's access to its transcriptional targets.

The cellular protein Kruppel-associated box domain-associated protein-1 (KAP-1) regulates formation of heterochromatin, and was found associated with most KSHV productive cycle promoters during latency (Chang et al., 2009). During reactivation, KAP-1 dissociated from promoters, concomitant with K-Rta DNA binding. KAP-1 knockdown induced reactivation (Chang et al., 2009).

The cellular protein high mobility group protein (HMG) B1 binds and bends DNA, and enhances K-Rta DNA binding. The effect of HMGB1 is greater for low affinity K-Rta binding sites (Song et al., 2004). Ectopic expression of HMGB1 enhanced KRta transactivation of the PAN, kaposin, vIL-6, Mta, and K-Rta promoters (Song et al., 2004; Harrison and Whitehouse, 2008).

\section{Rta COUNTERACTS ITS NEGATIVE REGULATORS}

K-Rta encodes a ubiquitin E3 ligase activity that regulates KSHV reactivation by targeting transcriptional repressors of K-Rta transactivation (Yu et al., 2005; Yang et al., 2008). K-Rta contains a Cys/His-Rich domain reminiscent of catalytic regions of E3 ligases (Figure 1). K-Rta targets the cellular protein IRF-7 for polyubiquitylation and degradation in a proteasome and Cys/Hisdependent manner (Yu et al., 2005). IRF-7 binds directly to K-Rta aa 273-544 (Yu et al., 2005; Figure 1).

K-Rta similarly targets the cellular protein K-RBP for degradation (Yang et al., 2008), but also requires K-Rta aa 621-641 for this effect (Figure 1). Poly-ubiquitylation by K-Rta corresponds with its ability to counteract transcriptional repression mediated by K-RBP. K-Rta expression is also associated with reduction of K-bZIP, LANA, and NF-kB p65 protein levels in 293 cells. Proteasome inhibitors reduced Rta-stimulated reactivation of KSHV in PEL cells. While Rta also autoregulates its own degradation by self-ubiquitylation (Yu et al., 2005), the biological significance has not been directly tested.

\section{CONCLUSION AND PERSPECTIVES SUMMARY}

Small DNA tumor viruses transform cells in non-productive, dead-end infections. The large DNA tumor virus KSHV establishes latent, or non-productive infections, which are reversible in a process called reactivation. In reactivation, KSHV genes are expressed in kinetically distinct cascades, leading to viral DNA replication and release of infectious virus. A single KSHV protein, $\mathrm{K}$-Rta, is necessary and sufficient to initiate the productive cascade of gene expression and the complete viral productive cycle. K-Rta is a nuclear, gene-specific transcriptional transactivator. K-Rta specifies promoters for transactivation by binding DNA directly and interacting with cellular DBPs.

Reactivation of KSHV from latency requires the cellular protein RBP-Jk to specify many of K-Rta's transcriptional targets. Thus, $\mathrm{K}$-Rta transactivation of KSHV promoters is either RBP-Jk independent or RBP-Jk-dependent. K-Rta transactivates RBP-Jk independent promoters by binding to a short, conserved, high affinity
K-Rta binding site (Figure 2). K-Rta transactivates the RBP-Jkdependent promoter, Mta, by binding to an extended element that contains multiple copies of a repetitive CANT DNA sequence, and stimulating RBP-Jk DNA binding (Figure 4A). CANT repeats are found in proximity to many RBP-Jk sites across the KSHV genome, and K-Rta stimulates RBP-Jk DNA binding broadly, but specifically, to the KSHV genome. A putative, working model based on genetic studies proposes that K-Rta stimulates RBP-Jk DNA binding by contacting CANT DNA repeats flanking an RBP-Jk site; biophysical investigations of this model are on-going. The inability of NICD1 and EBNA-2 to reactivate the complete KSHV productive cycle supports the existence of non-canonical regulation of RBP-Jk in KSHV infected cells.

Although K-Rta transactivates many of the KSHV promoters in transient transfections, and the KSHV genome contains at least 260 putative RBP-Jk binding sites, K-Rta only transactivates eight genes in the absence of de novo protein synthesis during reactivation. These data suggest that continued progression of reactivation is regulated after K-Rta expression.

Indeed, K-Rta appears to be a relatively inefficient reactivating switch, and can be regulated positively and negatively by many viral and cellular factors. One positively acting, K-Rta regulating factor is the essential KSHV protein Mta, which was identified as one of K-Rta's direct transcriptional targets. Mta cannot reactivate KSHV alone, but synergizes with K-Rta to induce complete reactivation. Single cell expression of Mta suggests that it is a commitment factor that commits a K-Rta expressing cell to complete reactivation. Mta has multiple, pleiotropic effects on gene expression. It is a cell and promoter specific transcriptional transactivator, and a transcript-specific post-transcriptional transactivator. Mta synergizes with K-Rta in a promoter specific manner, then might continue to transactivate post-transcriptionally by maintaining association with nascent RNAs. K-Rta also autoregulates its stability and DNA binding.

\section{CONTROVERSIES IN Rta PROMOTER SPECIFICATION}

Promoter specification by K-Rta has been controversial. Disagreements in published studies might be explained by technical variations in the strategies used to evaluate K-Rta DNA binding. Three examples of important technical differences might explain the divergent views. These disagreements raise some interesting biological questions about K-Rta.

In the first example, many reports agree that K-Rta binds to short segments of the Mta promoter directly (Duan et al., 2001; Lukac et al., 2001; Song et al., 2003; Carroll et al., 2006; Wen et al., 2009; Palmeri et al., 2011), yet other reports propose that K-Rta binds only indirectly to the Mta promoter (and others), by "piggybacking" onto DNA-bound RBP-Jk (Zhang et al., 1998; Sakakibara et al., 2001; Wang et al., 2003a,b, 2004a; Liang and Ganem, 2004; Chang et al., 2005c; Wang and Yuan, 2006). A recent publication demonstrated that K-Rta binds to a longer region of the Mta promoter then previously recognized. High affinity DNA binding (i.e., nM) of K-Rta requires seven copies of a CANT DNA element repeated over $62 \mathrm{bp}$ of the Mta promoter. Conversely, K-Rta binds DNAs containing three or fewer CANT repeats with 35-fold lower affinity. The earlier studies that concluded that K-Rta binds the Mta promoter 
indirectly all used similar short DNAs that contained three or fewer CANT repeats as K-Rta targets (Duan et al., 2001; Lukac et al., 2001; Song et al., 2003; Carroll et al., 2006; Wen et al., 2009). Thus, the Mta promoter is not an indirect target of $\mathrm{K}$ Rta, but rather, a high affinity RBP-Jk-dependent target. Biologically, the data suggest that K-Rta's affinity will vary significantly for natural promoters that contain varying numbers of CANT repeats.

In the second example, the choice of K-Rta expression system has led to disagreements regarding K-Rta's promoter specificity. In vitro, K-Rta only binds to Mta promoter DNAs when made in bacteria (Duan et al., 2001; Lukac et al., 2001; Song et al., 2003; Carroll et al., 2006; Wen et al., 2009; Palmeri et al., 2011), but never when made in mammalian systems (Wang et al., 2003a,b; Chang et al., 2005c). These data suggest that mammalian modifications, or co-purifying proteins, inhibit DNA binding of K-Rta to the Mta promoter. Interestingly, even though K-Rta aa 1-272 made in bacteria is sufficient to bind to the Mta promoter, its specificity seems to be more stringent than FL K-Rta made in bacteria (Carroll et al., 2006).

In the third example, different consensus sequences for $\mathrm{K}$ Rta DNA binding have been derived from KSHV genome-wide screens. In one study, ChIP-on-ChIP of K-Rta aa 1-390 expressed in BCBL-1 cells was performed $6 \mathrm{~h}$ after TPA induction, and generated a 40-bp consensus binding sequence that was sometimes found in tandem repeats (Chen et al., 2009). In another study, in vitro binding of K-Rta aa 1-530 to 200-300 nt fragments of the KSHV genome generated an 8-bp consensus binding sequence (Ziegelbauer et al., 2006). Besides deriving different KRta binding sites, the studies shared only two significant hits. The use of different sources and lengths of K-Rta protein, and an in vivo or in vitro approach, likely led to the different conclusions of the two studies. A third study screened 83 putative KSHV promoters using ChIP of FL K-Rta expressed in BCBL-1 cells $12 \mathrm{~h}$ after inducing reactivation, and identified 22 significant promoter hits (Ellison et al., 2009). Only eight of these promoters were also identified in the ChIP-on-ChIP screen (Chen et al., 2009). However, this study demonstrated that the choice of time point following reactivation could dramatically influence the number of K-Rta targets identified (Ellison et al., 2009). Therefore, it is likely that different conclusions between the two ChIP approaches could be explained by time after stimulation of reactivation, the use of different lengths of K-Rta protein, and different antibodies.

Clearly, there is much to be learned about K-Rta promoter specification and how it is regulated. In fact, the potential exists for disparity in one additional aspect of K-Rta DNA binding: the interpretation of phenotypes of K-Rta N-terminal mutants. The minimal K-Rta DNA binding domain maps to aa 1-272 (Lukac et al., 2001; Carroll et al., 2006), and overlaps with nine domains attributed to other functions of K-Rta (Figure 1): homooligomerization (Bu et al., 2007), RBP-Jk binding (Liang et al., 2002), Oct-1 binding (Carroll et al., 2007), E3 Ubiquitin ligation (Yu et al., 2005; Yang et al., 2008), inhibiting p53 (Gwack et al., 2001), K-RBP binding and degradation (Wang et al., 2001b; Yang et al., 2008), C/EBP binding (Wang et al., 2003a), and TLE2 binding (He et al., 2010). In fact, two publications have described single N-terminal point mutants that affect both DNA binding and interactions with either Oct-1 or $\mathrm{C} / \mathrm{EBP} \alpha$ (Wang et al., 2003a; Carroll et al., 2007). Many of these other N-terminal KRta functions have the potential to influence K-Rta promoter specification. A full understanding of K-Rta promoter selectivity requires more refinement of the K-Rta structure/function map to establish whether these functions are co-regulated or separable.

\section{K-Rta PROMOTER SPECIFICATION AND PROGRESSION OF VIRAL REACTIVATION}

The conclusion that K-Rta is an inefficient switch protein is based on results of single cell assays. K-Rta expressing PEL cells appear to have two fates that can be distinguished by Mta expression (Palmeri et al., 2007). In K-Rta expressing cells that co-express Mta, viral reactivation proceeds down the entire lytic cascade to release mature virus (see top of Figure 6). K-Rta activates Mta transcription, then Mta protein cooperates with K-Rta to transactivate additional DE genes, driving progression of reactivation. However, most K-Rta expressing cells do not co-express Mta, and are observed during KSHV reactivation up to $72 \mathrm{~h}$ post-TPA addition or post-Rta vector transfection (Palmeri et al., 2007; see bottom of Figure 6). Finally, a third fate of K-Rta expressing cells is suggested by the observation that both K-Rta and Mta are genespecific transactivators: K-Rta expression accompanied by a class of DE genes that are transactivated by K-Rta in the absence of Mta expression (see middle of Figure 6).

The other positive and negative regulators of K-Rta function can be considered using the single cell paradigm illustrated in Figure 6. Factors that stimulate K-Rta transactivation would drive reactivation toward the productive pathway (upwards), while factors that inhibit or temper K-Rta function would drive reactivation toward the non-productive pathways (downwards).

\section{AUTOREGULATION OF K-Rta EXPRESSION}

Numerous positive and negative regulatory events control K-Rta expression, and the K-Rta dosage clearly is proportional to the magnitude of reactivation (Sun et al., 1998). The key regulatory event in the latent to lytic switch is initiation of K-Rta transcription, which is not covered in detail here due to limitations of the publication format. However, following its expression, K-Rta auto-transactivates ORF 50 transcription in collaboration with Mta, Oct-1, AP-1, C/EBP $\alpha, \mathrm{K}-\mathrm{RBP}, \mathrm{HMGB} 1$, and XBP-1s (Deng et al., 2000; Sakakibara et al., 2001; Wang et al., 2001b, 2003a,b, 2004a; Malik et al., 2004a; Wilson et al., 2007; Harrison and Whitehouse, 2008; Di Bartolo et al., 2009). K-Rta auto-transactivation is inhibited by both cellular and viral factors, including TLE2, NFkB, Oct-2, Hey1, and LANA. (Brown et al., 2003; Lan et al., 2004, 2005a; Yada et al., 2006; Di Bartolo et al., 2009; He et al., 2010). Oct-2 inhibits K-Rta activation of the K-Rta promoter by preventing DNA binding of Oct-1 and K-Rta to the K-Rta promoter (Di Bartolo et al., 2009). LANA binds to both K-Rta and RBP-Jk, and competes with Rta for binding to RBP-Jk (Lan et al., 2004, 2005a), resulting in reduction of productive replication. Interestingly, K-Rta activates Heyl and LANA, the latter in an RBP-Jkdependent fashion (Lan et al., 2005b; Yada et al., 2006), to establish negative-feedback loops on its expression. Therefore, expression 


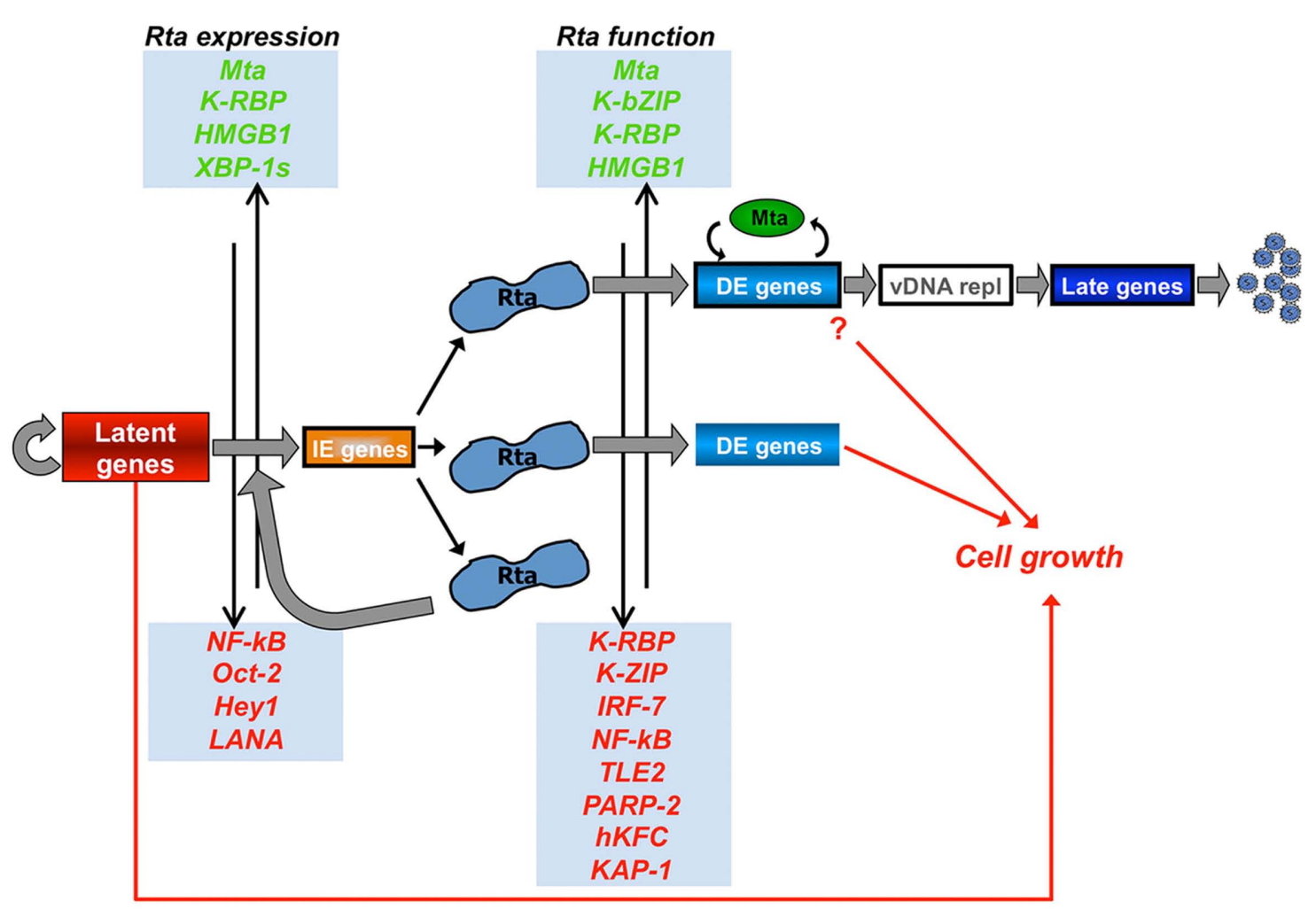

FIGURE 6 | Single cell model for progression of KSHV reactivation and its putative influence on cellular growth. As described in text, Rta expressing PEL cells are depicted as having three fates: complete reactivation (top), expression of a sub-set of DE genes without complete reactivation (middle), and completely abortive reactivation, in which K-Rta might feedback to activate itself (bottom). Modulators of K-Rta function that were discussed in the text are listed in the light blue boxes. Negative regulators (red text) might favor the non-productive reactivation fates, while positive regulators (green text) might favor the productive reactivation fate. DE oncogenes expressed in the top or middle fates, might cooperate with latent proteins to stimulate cell growth (red lines and text). IE, immediate early; DE, delayed early; vDNA repl, viral DNA replication. levels of K-Rta itself and of K-Rta's regulators of expression and function, work together to drive reactivation toward, or steer reactivation away from, the productive pathway (Figure 6). Any of K-Rta's regulatory factors that influence K-Rta's promoter specification could favor a specific path as illustrated, or a hypothetical "sub-path."

\section{PROGRESSION OF VIRAL REACTIVATION AND KSHV DISEASE}

Both latent and productive cycle genes are sufficient to transform cells when expressed alone, and animal models of KS demonstrate that productive cycle genes dramatically potentiate transformation driven by latent genes (reviewed in Martin and Gutkind, 2008; Mesri et al., 2010). This combinatorial effect would be consistent with the top and middle pathways of Figure 6. Most of KSHV's oncogenes are expressed in the DE class, suggesting that regulation of K-Rta's function can influence cell growth. Indeed, tissue culture models of KSHV infection support a role for K-Rta in contributing to growth of infected cells. In BC-1 PEL cells coinfected by KSHV and EBV, KSHV reactivation induced expression of the EBV oncogene latent membrane protein (LMP)-1 (Spadavecchia et al., 2010). Rta $\triangle$ STAD, but not the non-functional mutant Rta $\Delta$ STAD $\Delta$ LR, reduced growth of BC-1 cells within $24 \mathrm{~h}$ after transfection. As a control, Rta $\Delta$ STAD had no effect on growth of uninfected BL-41 cells, proving that its growth suppressing effect required cells to be infected by EBV and KSHV.

Since BC-1 cells were not uniformly transfected by the Rta $\triangle$ STAD expression vector, the data suggested that the BC1 cell population spontaneously expressed endogenous Rta in a non-synchronous and unsustained manner, which contributed to growth of BC-1 cells. In turn, LMP-1 is known to inhibit K-Rta expression and reactivation (Xu et al., 2007). Together, the data suggested a model in which spontaneously expressed Rta activated LMP-1, and LMP-1 provided a negative-feedback loop to promote a cellular environment in which Rta indirectly contributes to PEL growth without productively reactivating KSHV and lysing the infected cell.

In this system, K-Rta activated LMP-1 in an RBP-Jk-dependent manner, as if it was a KSHV DE gene (Spadavecchia et al., 2010). Although the role of KSHV oncogenes in Rta $\Delta$ STAD's growth effect in BC-1 cells has not been examined, it is reasonable to predict a similar contribution of Rta $\triangle$ STAD to the growth of singly infected, KSHV+ PEL cells. PEL cells characteristically display spontaneous reactivation in a small percentage of cells. Nonuniform expression of Rta $\triangle$ STAD in PEL cells inhibits spontaneous KSHV reactivation (Lukac et al., 1999). K-Rta transactivates many of the KSHV productive cycle, cellular survival and growth 
genes. In fact, three of these genes were identified as direct transcriptional targets of K-Rta: vIL-6, vIRF1, and K14 (Bu et al., 2008). $\mathrm{K} 14$ is co-transcribed with ORF 74, the viral G-protein coupled receptor (vGPCR; Kirshner et al., 1999). vGPCR negatively regulates Rta expression (Cannon et al., 2006) in a negative-feedback loop, similar to the effect of LMP-1 in co-infected cells.

The plethora of regulators of K-Rta function thus have the potential to influence cellular growth by modulating the progression of reactivation (Figure 6). In the examples of LMP-1 and vGPCR, the oncogenes downstream of K-Rta are themselves the negative regulators of K-Rta expression (i.e., the center pathway

in Figure 6). However, the models proposed in Figure 6 do not require the K-Rta regulators to be the actual oncogenes. Furthermore, expression of productive cycle oncogenes in the absence of K-Rta could also satisfy a model for unproductive reactivation (Chatterjee et al., 2002; Chang et al., 2005a). The applicability of these models to KSHV infection of endothelial cells is tempting but untested.

\section{ACKNOWLEDGMENTS}

The authors are supported by the NIH (R01AI078138 and T32CA134268).

cells. Proc. Natl. Acad. Sci. U.S.A. 102, 5570-5575.

Cannon, M., Cesarman, E., and Boshoff, C. (2006). KSHV G proteincoupled receptor inhibits lytic gene transcription in primary-effusion lymphoma cells via p21-mediated inhibition of Cdk2. Blood 107, 277-284.

Carroll, K. D., Bu, W., Palmeri, D. Spadavecchia, S., Lynch, S. J., Marras, S. A., Tyagi, S., and Lukac, D. M. (2006). Kaposi's sarcomaassociated herpesvirus lytic switch protein stimulates DNA binding of RBP-Jk/CSL to activate the Notch pathway. J. Virol. 80, 9697-9709.

Carroll, K. D., Khadim, F., Spadavecchia, S., Palmeri, D., and Lukac, D. M. (2007). Direct interactions of KSHV/HHV-8 ORF50/Rta protein with the cellular protein Octamer1 and DNA are critical for specifying transactivation of a delayedearly promoter and stimulating viral reactivation. J. Virol. 81, 8451-8467.

Cesarman, E., Moore, P. S., Rao, P. H., Inghirami, G., Knowles, D. M., and Chang, Y. (1995). In vitro establishment and characterization of two acquired immunodeficiency syndrome-related lymphoma cell lines (BC-1 and BC-2) containing Kaposi's sarcoma-associated herpesvirus-like (KSHV) DNA sequences. Blood 86, 2708-2714.

Chandriani, S., and Ganem, D. (2010). Array-based transcript profiling and limiting-dilution reverse transcription-PCR analysis identify additional latent genes in Kaposi's sarcoma-associated herpesvirus. J. Virol. 84, 5565-5573.

Chandriani, S., Xu, Y., and Ganem, D. (2010). The lytic transcriptome of Kaposi's sarcoma-associated herpesvirus reveals extensive transcription of noncoding regions, including regions antisense to important genes. J. Virol. 84, 7934-7942.

Chang, H., Dittmer, D. P., Chul, S. Y., Hong, Y., and Jung, J. U. (2005a). Role of Notch signal transduction in Kaposi's sarcoma-associated herpesvirus gene expression. J. Virol. 79, 14371-14382.

Chang, H., Gwack, Y., Kingston, D., Souvlis, J., Liang, X., Means, R. E., Cesarman, E., Hutt-Fletcher, L., and Jung, J. U. (2005b). Activation of $\mathrm{CD} 21$ and $\mathrm{CD} 23$ gene expression by Kaposi's sarcomaassociated herpesvirus RTA. J. Virol. 79, 4651-4663.

Chang, P. J., Shedd, D., and Miller, G. (2005c). Two subclasses of Kaposi's sarcoma-associated herpesvirus lytic cycle promoters distinguished by open reading frame 50 mutant proteins that are deficient in binding to DNA. J. Virol. 79, 8750-8763.

Chang, P. C., Fitzgerald, L. D., Hsia, D. A., Izumiya, Y., Wu, C. Y., Hsieh, W. P., Lin, S. F., Campbell, M. Lam, K. S., Luciw, P. A., Tepper, C. G., and Kung, H. J. (2011). Histone demethylase JMJD2A regulates Kaposi's sarcoma-associated herpesvirus replication and is targeted by a viral transcriptional factor. J. Virol. 85, 3283-3293.

Chang, P. C., Fitzgerald, L. D., Van Geelen, A., Izumiya, Y., Ellison, T. J., Wang, D. H., Ann, D. K., Luciw, P. A., and Kung, H. J. (2009). Kruppelassociated box domain-associated protein-1 as a latency regulator for Kaposi's sarcoma-associated herpesvirus and its modulation by the viral protein kinase. Cancer Res. 69, 5681-5689.

Chang, P. J., Boonsiri, J., Wang, S. S., Chen, L. Y., and Miller, G. (2010). Binding of RBP-Jkappa (CSL) protein to the promoter of the Kaposi's sarcoma-associated herpesvirus ORF47 (gL) gene is a critical but not sufficient determinant of transactivation by ORF50 protein. Virology 398, 38-48.

Chang, P. J., and Miller, G. (2004). Autoregulation of DNA binding and protein stability of Kaposi's sarcoma-associated herpesvirus ORF50 protein. J. Virol. 78, 10657-10673. 
Chang, P. J., Shedd, D., Gradoville, L., Cho, M. S., Chen, L. W., Chang, J., and Miller, G. (2002). Open reading frame 50 protein of Kaposi's sarcoma-associated herpesvirus directly activates the viral PAN and K12 genes by binding to related response elements. J. Virol. 76, 3168-3178.

Chang, P. J., Shedd, D., and Miller, G. (2008). A mobile functional region of Kaposi's sarcoma-associated herpesvirus ORF50 protein independently regulates DNA binding and protein abundance. J. Virol. 82, 9700-9716.

Chang, Y., and Moore, P. S. (2011). Merkel cell carcinoma: a virusinduced human cancer. Annu. Rev. Pathol. PMID: 21942528. [Epub ahead of print].

Chatterjee, M., Osborne, J., Bestetti, G., Chang, Y., and Moore, P. S. (2002). Viral IL-6-induced cell proliferation and immune evasion of interferon activity. Science 298, 1432-1435.

Chen, J., Ueka, K., Sakakibara, S., Okuno, T., and Yamanishi, K. (2000). Transcriptional regulation of the Kaposi's sarcoma-associated herpesvirus viral interferon regulatory factor gene. J. Virol. 74, 8623-8634.

Chen, J., Ye, F., Xie, J., Kuhne, K., and Gao, S. J. (2009). Genomewide identification of binding sites for Kaposi's sarcoma-associated herpesvirus lytic switch protein, RTA. Virology 386, 290-302.

Conrad, N. K. (2009). Posttranscriptional gene regulation in Kaposi's sarcoma-associated herpesvirus. Adv. Appl. Microbiol. 68, 241-261.

Damania, B., Jeong, J. H., Bowser, B. S., Dewire, S. M., Staudt, M. R., and Dittmer, D. P. (2004). Comparison of the Rta/Orf50 transactivator proteins of gamma-2-herpesviruses. $J$. Virol. 78, 5491-5499.

Deng, H., Young, A., and Sun, R. (2000). Auto-activation of the rta gene of human herpesvirus-8/Kaposi's sarcoma-associated herpesvirus. $J$. Gen. Virol. 81, 3043-3048.

Di Bartolo, D. L., Hyjek, E., Keller, S., Guasparri, I., Deng, H., Sun, R., Chadburn, A., Knowles, D. M., and Cesarman, E. (2009). Role of defective Oct- 2 and OCA-B expression in immunoglobulin production and Kaposi's sarcoma-associated herpesvirus lytic reactivation in primary effusion lymphoma. J. Virol. 83, 4308-4315.

Drexler, H., Uphoff, C., Gaidano, G., and Carbone, A. (1998). Lymphoma cell lines: in vitro models for the study of HHV-8+ primary effusion lymphomas (body cavity-based lymhomas). Leukemia 12, 1507-1517.

Duan, W., Wang, S., Liu, S., and Wood, C. (2001). Characterization of Kaposi's sarcoma-associated herpesvirus/human herpesvirus-8 ORF57 promoter. Arch. Virol. 146, 403-413.

Ellison, T. J., Izumiya, Y., Izumiya, C., Luciw, P. A., and Kung, H. J. (2009). A comprehensive analysis of recruitment and transactivation potential of K-Rta and K-bZIP during reactivation of Kaposi's sarcomaassociated herpesvirus. Virology 387, 76-88.

Fakhari, F. D., and Dittmer, D. P. (2002). Charting latency transcripts in Kaposi's sarcoma-associated herpesvirus by whole-genome realtime quantitative PCR. J. Virol. 76, 6213-6223.

Ganem, D. (2007). "Kaposi's sarcomaassociated herpesvirus," in Fields Virology, eds D. M. Knipe and P. M. Howley (Philadelphia: Lippincott Williams and Wilkins), 2848-2888.

Gradoville, L., Gerlach, J., Grogan, E., Shedd, D., Nikiforow, S., Metroka, C., and Miller, G. (2000). Kaposi's sarcoma-associated herpesvirus open reading frame $50 /$ Rta protein activates the entire lytic cycle in the HH-B2 primary effusion lymphoma cell line. J. Virol. 74, 6207-6212.

Grundhoff, A., and Ganem, D. (2004). Inefficient establishment of KSHV latency suggests an additional role for continued lytic replication in Kaposi sarcoma pathogenesis. J. Clin. Invest. 113, 124-136.

Gunther, T., and Grundhoff, A. (2010). The epigenetic landscape of latent Kaposi sarcomaassociated herpesvirus genomes. PLoS Pathog. 6, e1000935. doi:10.1371/journal.ppat.1000935

Gupta, A. K., Ruvolo, V., Patterson, C., and Swaminathan, S. (2000). The human herpesvirus 8 homolog of Epstein-Barr virus SM protein (KS$\mathrm{SM}$ ) is a posttranscriptional activator of gene expression. J. Virol. 74, 1038-1044.

Gwack, Y., Hwang, S., Byun, H., Lim, C., Kim, J. W., Choi, E. J., and Choe, J. (2001). Kaposi's sarcomaassociated herpesvirus open reading frame 50 represses p53-induced transcriptional activity and apoptosis. J. Virol. 75, 6245-6248.

Gwack, Y., Hwang, S., Lim, C., Won, Y. S., Lee, C. H., and Choe, J. (2002). Kaposi's sarcoma-associated herpesvirus open reading frame 50 stimulates the transcriptional activity of STAT3. J. Biol. Chem. 277, 6438-6442.
Gwack, Y., Nakamura, H., Lee, S. H., Souvlis, J., Yustein, J. T., Gygi, S., Kung, H. J., and Jung, J. U. (2003) Poly(ADP-ribose) polymerase 1 and Ste20-like kinase hKFC act as transcriptional repressors for gamma2 herpesvirus lytic replication. Mol. Cell. Biol. 23, 8282-8294.

Han, Z., and Swaminathan, S. (2006). Kaposi's sarcoma-associated herpesvirus lytic gene ORF57 is essential for infectious virion production. $J$. Virol. 80, 5251-5260.

Harrison, S. M., and Whitehouse, A. (2008). Kaposi's sarcoma-associated herpesvirus (KSHV) Rta and cellular HMGB1 proteins synergistically transactivate the KSHV ORF50 promoter. FEBS Lett. 582, 3080-3084.

He, Z., Liu, Y., Liang, D., Wang, Z., Robertson, E. S., and Lan, K. (2010). Cellular corepressor TLE2 inhibits replication-and-transcriptionactivator-mediated transactivation and lytic reactivation of Kaposi's sarcoma-associated herpesvirus. J. Virol. 84, 2047-2062.

Izumiya, Y., Izumiya, C., Hsia, D., Ellison, T. J., Luciw, P. A., and Kung, H. J. (2009). NF-kappaB serves as a cellular sensor of Kaposi's sarcomaassociated herpesvirus latency and negatively regulates K-Rta by antagonizing the RBP-Jkappa coactivator. J. Virol. 83, 4435-4446.

Jackson, B. R., Boyne, J. R., Noerenberg, M., Taylor, A., Hautbergue, G. M., Walsh, M. J., Wheat, R., Blackbourn, D. J., Wilson, S. A., and Whitehouse, A. (2011). An interaction between KSHV ORF57 and UIF provides mRNA-adaptor redundancy in herpesvirus intronless mRNA export. PLoS Pathog. 7, e1002138. doi:10.1371/journal.ppat.1002138

Jenner, R., Alba, M., Boshoff, C., and Kellam, P. (2001). Kaposi's sarcomaassociated herpesvirus latent and lytic gene expression as revealed by DNA arrays. J. Virol. 75, 891-902.

Kang, H., Wiedmer, A., Yuan, Y., Robertson, E., and Lieberman, P. M. (2011a). Coordination of KSHV latent and lytic gene control by CTCF-cohesin mediated chromosome conformation. PLoS Pathog. 7, e1002140. doi:10.1371/journal.ppat.1002140

Kang, J. G., Pripuzova, N., Majerciak, V., Kruhlak, M., Le, S. Y., and Zheng, Z. M. (2011b). Kaposi's sarcoma-associated herpesvirus ORF57 promotes escape of viral and human interleukin- 6 from microRNA-mediated suppression. $J$ Virol. 85, 2620-2630.
Kato-Noah, T., Xu, Y., Rossetto, C. C., Colletti, K., Papouskova, I., and Pari, G. S. (2007). Overexpression of the kaposi's sarcomaassociated herpesvirus transactivator K-Rta can complement a KbZIP deletion BACmid and yields an enhanced growth phenotype. $J$. Virol. 81, 13519-13532.

Kieff, E., and Rickinson, A. (2007). "Epstein-barr virus and its replication," in Fields Virology, 5th Edn. eds D. M. Knipe and P. M. Howley (Philadelphia, PA: Lippincott Williams and Wilkins), 2603-2654.

Kirshner, J. R., Lukac, D. M., Chang, J., and Ganem, D. (2000). Kaposi's sarcoma-associated herpesvirus open reading frame 57 encodes a posttranscriptional regulator with multiple distinct activities. J. Virol. 74, 3586-3597.

Kirshner, J. R., Staskus, K., Haase, A., Lagunoff, M., and Ganem, D. (1999). Expression of the open reading frame 74 (G-protein-coupled receptor) gene of Kaposi's sarcoma (KS)associated herpesvirus; implications for KS pathogenesis. J. Virol. 73, 6006-6014.

Lan, K., Kuppers, D. A., and Robertson, E. S. (2005a). Kaposi's sarcomaassociated herpesvirus reactivation is regulated by interaction of latency-associated nuclear antigen with recombination signal sequence-binding protein Jkappa, the major downstream effector of the Notch signaling pathway. J. Virol. 79, 3468-3478.

Lan, K., Kuppers, D. A., Verma, S. C., Sharma, N., Murakami, M., and Robertson, E. S. (2005b). Induction of Kaposi's sarcoma-associated herpesvirus latency-associated nuclear antigen by the lytic transactivator RTA: a novel mechanism for establishment of latency. J. Virol. 79, 7453-7465.

Lan, K., Kuppers, D. A., Verma, S. C., and Robertson, E. S. (2004). Kaposi's sarcoma-associated herpesvirusencoded latency-associated nuclear antigen inhibits lytic replication by targeting Rta: a potential mechanism for virus-mediated control of latency. J. Virol. 78, 6585-6594.

Liang, Y., Chang, J., Lynch, S., Lukac, D. M., and Ganem, D. (2002). The lytic switch protein of KSHV activates gene expression via functional interaction with RBP-Jk, the target of the Notch signaling pathway. Genes Dev. 16, 1977-1989.

Liang, Y., and Ganem, D. (2003). Lytic but not latent infection by Kaposi's sarcoma-associated herpesvirus requires host CSL protein, 
the mediator of Notch signaling. Proc. Natl. Acad. Sci. U.S.A. 100, 8490-8495.

Liang, Y., and Ganem, D. (2004). RBP$\mathrm{J}$ (CSL) is essential for activation of the K14/vGPCR promoter of Kaposi's sarcoma-associated herpesvirus by the lytic switch protein RTA. J. Virol. 78, 6818-6826.

Liao, W., Tang, Y., Kuo, Y. L., Liu, B. Y., Xu, C. J., and Giam, C. Z. (2003a). Kaposi's sarcomaassociated herpesvirus/human herpesvirus 8 transcriptional activator Rta is an oligomeric DNA-binding protein that interacts with tandem arrays of phased A/T-trinucleotide motifs. J. Virol. 77, 9399-9411.

Liao, W., Tang, Y., Lin, S. F., Kung, H. J., and Giam, C. Z. (2003b). K-bZIP of Kaposi's sarcomaassociated herpesvirus/human herpesvirus 8 (KSHV/HHV-8) binds KSHV/HHV-8 Rta and represses Rta-mediated transactivation. J. Virol. 77, 3809-3815.

Liu, Y., Cao, Y., Liang, D., Gao, Y., Xia, T., Robertson, E. S., and Lan, K. (2008). Kaposi's sarcoma-associated herpesvirus RTA activates the processivity factor ORF59 through interaction with RBP-Jkappa and a cis-acting RTA responsive element. Virology 380, 264-275.

Lu, M., Suen, J., Frias, C., Pfeiffer, R., Tsai, M. H., Chuang, E., and Zeichner, S. L. (2004). Dissection of the Kaposi's sarcoma-associated herpesvirus gene expression program by using the viral DNA replication inhibitor cidofovir. J. Virol. 78, 13637-13652.

Lukac, D., Garibyan, L., Kirshner, J., Palmeri, D., and Ganem, D. (2001). DNA binding by the Kaposi's sarcoma-associated herpesvirus lytic switch protein is necessary for transcriptional activation of two viral delayed early promoters. J. Virol. 75, 6786-6799.

Lukac, D. M., Kirshner, J. R., and Ganem, D. (1999). Transcriptional activation by the product of open reading frame 50 of Kaposi's sarcoma-associated herpesvirus is required for lytic viral reactivation in B cells. J. Virol. 73, 9348-9361.

Lukac, D. M., Renne, R., Kirshner, J. R., and Ganem, D. (1998). Reactivation of Kaposi's sarcoma-associated herpesvirus infection from latency by expression of the ORF 50 transactivator, a homolog of the EBV R protein. Virology 252, 304-312.

Majerciak, V., Pripuzova, N., Mccoy, J. P., Gao, S. J., and Zheng, Z. M. (2006a). Targeted disruption of KSHV ORF57 in the viral genome is detrimental for the expression of ORF59, K8\{alpha\}, and K8.1 and the production of infectious virus. J. Virol. 81, 1062-1071.

Majerciak, V., Yamanegi, K., Nie, S. H., and Zheng, Z. M. (2006b). Structural and functional analyses of Kaposi sarcoma-associated herpesvirus ORF57 nuclear localization signals in living cells. J. Biol. Chem. 281, 28365-28378.

Majerciak, V., Yamanegi, K., and Zheng, Z. M. (2006c). Gene structure and expression of Kaposi's sarcomaassociated herpesvirus ORF56, ORF57, ORF58, and ORF59. J. Virol. 80, 11968-11981.

Majerciak, V., Pripuzova, N., Mccoy, J. P., Gao, S. J., and Zheng, Z. M. (2007). Targeted disruption of Kaposi's sarcoma-associated herpesvirus ORF57 in the viral genome is detrimental for the expression of ORF59, K8alpha, and K8.1 and the production of infectious virus. $J$. Virol. 81, 1062-1071.

Majerciak, V., Uranishi, H., Kruhlak, M., Pilkington, G. R., Massimelli, M. J., Bear, J., Pavlakis, G. N., Felber, B. K., and Zheng, Z. M. (2011). Kaposi's sarcomaassociated herpesvirus ORF57 interacts with cellular RNA export cofactors RBM15 and OTT3 to promote expression of viral ORF59. J. Virol. 85, 1528-1540.

Majerciak, V., Yamanegi, K., Allemand, E., Kruhlak, M., Krainer, A. R., and Zheng, Z. M. (2008). Kaposi's sarcoma-associated herpesvirus ORF57 functions as a viral splicing factor and promotes expression of intron-containing viral lytic genes in spliceosomemediated RNA splicing. J. Virol. 82, 2792-2801.

Majerciak, V., and Zheng, Z. M. (2009). Kaposi's sarcoma-associated herpesvirus ORF57 in viral RNA processing. Front. Biosci. 14, 1516-1528.

Malik, P., Blackbourn, D. J., Cheng, M. F., Hayward, G. S., and Clements, J. B. (2004a). Functional co-operation between the Kaposi's sarcomaassociated herpesvirus ORF57 and ORF50 regulatory proteins. J. Gen. Virol. 85, 2155-2166.

Malik, P., Blackbourn, D. J., and Clements, J. B. (2004b). The evolutionarily conserved Kaposi's sarcoma-associated herpesvirus ORF57 protein interacts with REF protein and acts as an RNA export factor. J. Biol. Chem. 279, 33001-33011.

Martin, D., and Gutkind, J. S. (2008). Human tumor-associated viruses and new insights into the molecular mechanisms of cancer. Oncogene 27(Suppl. 2), S31-S42.

Massimelli, M. J., Kang, J. G., Majerciak, V., Le, S. Y., Liewehr, D. J., Steinberg, S. M., and Zheng, Z. M. (2011). Stability of a long noncoding viral RNA depends on a 9-nt core element at the RNA $5^{\prime}$ end to interact with viral ORF57 and cellular PABPC1. Int. J. Biol. Sci. 7, 1145-1160.

Mesri, E. A., Cesarman, E., and Boshoff, C. (2010). Kaposi's sarcoma and its associated herpesvirus. Nat. Rev Cancer 10, 707-719.

Moody, C. A., and Laimins, L. A. (2010). Human papillomavirus oncoproteins: pathways to transformation. Nat. Rev. Cancer 10, 550-560.

Moore, P. S., Gao, S. J., Dominguez, G. Cesarman, E., Lungu, O., Knowles, D. M., Garber, R., Pellett, P. E. Mcgeoch, D. J., and Chang, Y. (1996). Primary characterization of a herpesvirus agent associated with Kaposi's sarcomae. J. Virol. 70, 549-558.

Nakamura, H., Lu, M., Gwack, Y., Souvlis, J., Zeichner, S. L., and Jung, J. U. (2003). Global changes in Kaposi's sarcoma-associated virus gene expression patterns following expression of a tetracyclineinducible Rta transactivator. J. Virol. 77, 4205-4220.

Nekorchuk, M., Han, Z., Hsieh, T. T., and Swaminathan, S. (2007). Kaposi's sarcoma-associated herpesvirus ORF57 protein enhances mRNA accumulation independently of effects on nuclear RNA export. $J$. Virol. 81, 9990-9998.

Nishimura, K., Ueda, K., Guwanan, E., Sakakibara, S., Do, E., Osaki, E., Yada, K., Okuno, T., and Yamanishi, K. (2004). A posttranscriptional regulator of Kaposi's sarcomaassociated herpesvirus interacts with RNA-binding protein PCBP1 and controls gene expression through the IRES. Virology 325 , 364-378.

Osada, S., Yamamoto, H., Nishihara, T., and Imagawa, M. (1996). DNA binding specificity of the CCAAT/enhancer-binding protein transcription factor family. J. Biol. Chem. 271, 3891-3896.

Palmeri, D., Carroll, K. D., GonzalezLopez, O., and Lukac, D. M. (2011). Kaposi's sarcoma-associated herpesvirus Rta tetramers make highaffinity interactions with repetitive DNA elements in the Mta promoter to stimulate DNA binding of RBPJk/CSL. J. Virol. 85, 11901-11915.

Palmeri, D., Spadavecchia, S., Carroll, K., and Lukac, D. M. (2007). Promoter and cell-specific transcriptional activation by the Kaposi's sarcoma-associated herpesvirus ORF57/Mta protein. $J$. Virol. 81, 13299-13314.

Paulose-Murphy, M., Ha, N.-K., Xiang, C., Chen, Y., Gillim, L., Yarchoan, R., Meltzer, P., Bittner, M., Trent, J., and Zeichner, S. (2001). Transcription program of Human herpesvirus 8 (Kaposi's sarcoma-associated herpesvirus). J. Virol. 75, 4843-4853.

Persson, L. M., and Wilson, A. C. (2010). Wide-scale use of Notch signaling factor CSL/RBP-Jkappa in RTA-mediated activation of Kaposi's sarcoma-associated herpesvirus lytic genes. J. Virol. 84, 1334-1347.

Renne, R., Blackbourn, D., Whitby, D., Levy, J., and Ganem, D. (1998). Limited transmission of Kaposi's sarcoma-associated herpesvirus in cultured cells. J. Virol. 72, 5182-5188

Renne, R., Zhong, W., Herndier, B., Mcgrath, M., Abbey, N., Kedes, D., and Ganem, D. (1996). Lytic growth of Kaposi's sarcoma-associated herpesvirus (human herpesvirus 8 ) in culture. Nat. Med. 2, 342-346.

Rickinson, A., and Kieff, E. (2007). "Epstein-barr virus," in Fields Virology, eds D. M. Knipe and P. M. Howley (Philadelphia, PA: Lippincott Williams and Wilkins), 2656-2700.

Rimessi, P., Bonaccorsi, A., Sturzl, M., Fabris, M., Brocca-Cofano, E., Caputo, A., Melucci-Vigo, G., Falchi, M., Cafaro, A., Cassai, E., Ensoli, B., and Monini, P. (2001). Transcription pattern of human herpesvirus 8 open reading frame $\mathrm{K} 3$ in primary effusion lymphoma and Kaposi's sarcoma. J. Virol. 75, 7161-7174.

Roan, F., Inoue, N., and Offermann, M. K. (2002). Activation of cellular and heterologous promoters by the human herpesvirus 8 replication and transcription activator. Virology 301 , 293-304.

Russo, J. J., Bohenzky, R. A., Chien, M. C., Chen, J., Yan, M., Maddalena, D., Parry, J. P., Peruzzi, D., Edelman, I. S., Chang, Y., and Moore, P. S. (1996). Nucleotide sequence of the Kaposi sarcoma-associated herpesvirus (HHV8). Proc. Natl. Acad. Sci. U.S.A. 93, 14862-14867.

Sahin, B. B., Patel, D., and Conrad, N. K. (2010). Kaposi's sarcoma-associated herpesvirus ORF57 protein binds and protects a nuclear noncoding RNA from cellular RNA decay pathways. PLoS Pathog. 6, e1000799. doi:10.1371/journal.ppat.1000799

Sakakibara, S., Ueda, K., Chen, J., Okuno, T., and Yamanishi, K. (2001). Octamer-binding sequence is a key element for the 
autoregulation of Kaposi's sarcomaassociated herpesvirus ORF50/Lyta gene expression. J. Virol. 75, 6894-6800.

Samols, M. A., Hu, J., Skalsky, R. L., and Renne, R. (2005). Cloning and identification of a microRNA cluster within the latency-associated region of Kaposi's sarcomaassociated herpesvirus. J. Virol. 79, 9301-9305.

Sarid, R., Flore, O., Bohenzky, R. A., Chang, Y., and Moore, P. S. (1998). Transcription mapping of the Kaposi's sarcoma-associated herpesvirus (human herpesvirus 8) genome in a body cavity-based lymphoma cell line (BC-1). J. Virol. 72, 1005-1012.

Seaman, W., Ye, D., Wang, R., Hale, E., Weisse, M., and Quinlivan, E. (1999). Gene expression from the ORF50/K8 region of Kaposi's sarcoma-associated herpesvirus. Virology 263, 436-449.

Sei, E., and Conrad, N. K. (2011). Delineation of a core RNA element required for Kaposi's sarcomaassociated herpesvirus ORF57 binding and activity. Virology 419, 107-116.

Song, M., Brown, H., Wu, T.-T., and Sun, R. (2001). Transcription activation of polyadenylated nuclear RNA by Rta in human herpesvirus 8/Kaposi's sarcoma-associated herpesvirus. $J$. Virol. 75, 3129-3140.

Song, M. J., Deng, H., and Sun, R. (2003). Comparative study of regulation of RTA-responsive genes in Kaposi's sarcoma-associated herpesvirus/human herpesvirus $8 . \mathrm{J}$. Virol. 77, 9451-9462.

Song, M. J., Hwang, S., Wong, W., Round, J., Martinez-Guzman, D., Turpaz, Y., Liang, J., Wong, B., Johnson, R. C., Carey, M., and Sun, R. (2004). The DNA architectural protein HMGB1 facilitates RTAmediated viral gene expression in gamma-2 herpesviruses. J. Virol. 78, 12940-12950.

Song, M. J., Li, X., Brown, H. J., and Sun, R. (2002). Characterization of interactions between RTA and the promoter of polyadenylated nuclear RNA in Kaposi's sarcoma-associated herpesvirus/human herpesvirus 8. J. Virol. 76, 5000-5013.

Spadavecchia, S., Gonzalez-Lopez, O., Carroll, K. D., Palmeri, D, and Lukac, D. M. (2010). Convergence of Kaposi's sarcoma-associated herpesvirus reactivation with EpsteinBarr virus latency and cellular growth mediated by the notch signaling pathway in coinfected cells. $J$. Virol. 84, 10488-10500.
Staskus, K. A., Sun, R., Miller, G., Racz, P., Jaslowski, A., Metroka, C., Brett-Smith, H., and Haase, A. T. (1999). Cellular tropism and viral interleukin-6 expression distinguish human herpesvirus 8 involvement in Kaposi's sarcoma, primary effusion lymphoma, and multicentric Castleman's disease. J. Virol. 73, 4181-4187.

Staskus, K. A., Zhong, W., Gebhard, K., Herndier, B., Wang, H., Renne, R., Beneke, J., Pudney, J., Anderson, D. J., Ganem, D., and Haase, A. T. (1997). Kaposi's sarcomaassociated herpesvirus gene expression in endothelial (spindle) tumor cells. J. Virol. 71, 715-719.

Sun, R., Lin, S., Gradoville, L., and Miller, G. (1996). Polyadenylated nuclear RNA encoded by Kaposi's sarcoma-associated herpesvirus. Proc. Natl. Acad. Sci. U.S.A. 93, 11883-11888.

Sun, R., Lin, S. F., Gradoville, L., Yuan, Y., Zhu, F., and Miller, G. (1998). A viral gene that activates lytic cycle expression of Kaposi's sarcoma-associated herpesvirus. Proc. Natl. Acad. Sci. U.S.A. 95, 10866-10871.

Sun, R., Lin, S.-F., Staskus, K., Gradoville, L., Grogan, E., Haase, A., and Miller, G. (1999). Kinetics of Kaposi's sarcoma-associated herpesvirus gene expression. J. Virol. $73,2232-2242$.

Swaminathan, S. (2005). Posttranscriptional gene regulation by gamma herpesviruses. J. Cell. Biochem. 95, 698-711.

Tanigaki, K., and Honjo, T. (2010). Two opposing roles of RBP-J in Notch signaling. Curr. Top. Dev. Biol. 92, 231-252.

Taylor, A., Jackson, B. R., Noerenberg, M., Hughes, D. J., Boyne, J. R., Verow, M., Harris, M., and Whitehouse, A. (2011). Mutation of a C-terminal motif affects Kaposi's sarcoma-associated herpesvirus ORF57 RNA binding, nuclear trafficking, and multimerization. J. Virol. 85, 7881-7891.

Toth, Z., Maglinte, D. T., Lee, S. H., Lee, H. R., Wong, L. Y., Brulois, K. F., Lee, S., Buckley, J. D., Laird, P. W., Marquez, V. E., and Jung, J. U. (2010). Epigenetic analysis of KSHV latent and lytic genomes. PLoS Pathog. 6, el001013. doi:10.1371/journal.ppat.1001013

Ueda, K., Ishikawa, K., Nishimura, K., Sakakibara, S., Do, E., and Yamanishi, K. (2002). Kaposi's sarcoma-associated herpesvirus (human herpesvirus 8) replication and transcription factor activates the K9 (vIRF) gene through two distinct cis elements by a non-DNAbinding mechanism. J. Virol. 76 12044-12054.

Vieira, J., and O'Hearn, P. M. (2004). Use of the red fluorescent protein as a marker of Kaposi's sarcomaassociated herpesvirus lytic gene expression. Virology 325, 225-240.

Wang, J., Zhang, J., Zhang, L., Harrington, W. Jr., West, J. T., and Wood, C. (2005). Modulation of human herpesvirus 8/Kaposi's sarcomaassociated herpesvirus replication and transcription activator transactivation by interferon regulatory factor 7. J. Virol. 79, 2420-2431.

Wang, S., Liu, S., Wu, M., Geng, Y., and Wood, C. (2001a). Kaposi's sarcomaassociated herpesvirus/human herpesvirus-8 ORF50 gene product contains a potent C-terminal activation domain which activates gene expression via a specific target sequence. Arch. Virol. 146 , 1415-1426.

Wang, S., Liu, S., Wu, M. H., Geng, Y., and Wood, C. (2001b). Identification of a cellular protein that interacts and synergizes with the RTA (ORF50) protein of Kaposi's sarcoma-associated herpesvirus in transcriptional activation. J. Virol. 75, 11961-11973.

Wang, S. E., Wu, F. Y., Chen, H., Shamay, M., Zheng, Q., and Hayward, G. S. (2004a). Early activation of the Kaposi's sarcomaassociated herpesvirus RTA, RAP, and MTA promoters by the tetradecanoyl phorbol acetateinduced AP1 pathway. J. Virol. 78, 4248-4267.

Wang, Y., Li, H., Chan, M. Y., Zhu, F. X., Lukac, D. M., and Yuan, Y. (2004b). Kaposi's sarcoma-associated herpesvirus ori-Lyt-dependent DNA replication: cis-acting requirements for replication and ori-Lytassociated RNA transcription. J. Virol. 78, 8615-8629.

Wang, S. E., Wu, F. Y., Fujimuro, M., Zong, J., Hayward, S. D., and Hayward, G. S. (2003a). Role of CCAAT/enhancer-binding protein alpha (C/EBPalpha) in activation of the Kaposi's sarcoma-associated herpesvirus (KSHV) lytic-cycle replication-associated protein (RAP) promoter in cooperation with the KSHV replication and transcription activator (RTA) and RAP. J. Virol. 77, 600-623.

Wang, S. E., Wu, F. Y., Yu, Y. and Hayward, G. S. (2003b). CCAAT/enhancer-binding protein-alpha is induced during the early stages of Kaposi's sarcoma-associated herpesvirus
(KSHV) lytic cycle reactivation and together with the KSHV replication and transcription activator (RTA) cooperatively stimulates the viral RTA, MTA, and PAN promoters. $J$. Virol. 77, 9590-9612.

Wang, S. S., Chen, L. W., Chen, L. Y., Tsai, H. H., Shih, Y. C., Yang, C. T., and Chang, P. J. (2010). Transcriptional regulation of the ORF61 and ORF60 genes of Kaposi's sarcomaassociated herpesvirus. Virology 397, 311-321.

Wang, Y., Tang, Q., Maul, G. G., and Yuan, Y. (2006). Kaposi's sarcoma-associated herpesvirus oriLyt-dependent DNA replication: dual role of replication and transcription activator. J. Virol. 80, 12171-12186.

Wang, Y., and Yuan, Y. (2006). Essential role of RBP-Jkappa in activation of the K8 delayed-early promoter of Kaposi's sarcoma-associated herpesvirus by ORF50/RTA. Virology $359,19-27$.

Wen, H. J., Minhas, V., and Wood, C. (2009). Identification and characterization of a new Kaposi's sarcomaassociated herpesvirus replication and transcription activator (RTA)responsive element involved in RTAmediated transactivation. J. Gen Virol. 90, 944-953.

Wilson, S. J., Tsao, E. H., Webb, B. L., Ye, H., Dalton-Griffin, L., Tsantoulas, C., Gale, C. V., Du, M. Q., Whitehouse, A., and Kellam, P. (2007). X box binding protein XBP-1s transactivates the Kaposi's sarcoma-associated herpesvirus (KSHV) ORF50 promoter, linking plasma cell differentiation to KSHV reactivation from latency. $J$. Virol. 81, 13578-13586.

Xu, D., Coleman, T., Zhang, J., Fagot, A., Kotalik, C., Zhao, L. Trivedi, P., Jones, C., and Zhang, L. (2007). Epstein-barr virus inhibits Kaposi's sarcoma-associated herpesvirus lytic replication in primary effusion lymphomas. J. Virol. 81 , 6068-6078.

Xu, Y., Aucoin, D. P., Huete, A. R., Cei, S. A., Hanson, L. J., and Pari, G. S. (2005). A Kaposi's sarcomaassociated herpesvirus/human herpesvirus 8 ORF50 deletion mutant is defective for reactivation of latent virus and DNA replication. J. Virol. 79, 3479-3487.

$\mathrm{Xu}$, Y., and Ganem, D. (2010). Making sense of antisense: seemingly noncoding RNAs antisense to the master regulator of Kaposi's sarcoma-associated herpesvirus lytic replication do not regulate that transcript but serve as mRNAs 
encoding small peptides. J. Virol. 84, 5465-5475.

Yada, K., Do, E., Sakakibara, S., Ohsaki, E., Ito, E., Watanabe, S., and Ueda, K. (2006). KSHV RTA induces a transcriptional repressor, HEY1 that represses rta promoter. Biochem. Biophys. Res. Commun. $345,410-418$.

Yang, Z., Wen, H. J., Minhas, V., and Wood, C. (2009). The zinc finger DNA-binding domain of K-RBP plays an important role in regulating Kaposi's sarcoma-associated herpesvirus RTA-mediated gene expression. Virology 391, 221-231.

Yang, Z., and Wood, C. (2007). The transcriptional repressor K-RBP modulates RTA-mediated transactivation and lytic replication of Kaposi's sarcoma-associated herpesvirus. $J$. Virol. 81, 6294-6306.

Yang, Z., Yan, Z., and Wood, C. (2008). Kaposi's sarcoma-associated herpesvirus transactivator RTA promotes degradation of the repressors to regulate viral lytic replication. $J$. Virol. 82, 3590-3603.

Yu, Y., Wang, S. E., and Hayward, G. S. (2005). The KSHV immediate-early transcription factor RTA encodes ubiquitin E3 ligase activity that targets IRF7 for proteosome-mediated degradation. Immunity 22, 59-70.

Zhang, J., Wang, J., Wood, C., Xu, D., and Zhang, L. (2005). Kaposi's sarcomaassociated herpesvirus/human herpesvirus 8 replication and transcription activator regulates viral and cellular genes via interferon-stimulated response elements. J. Virol. 79, 5640-5652.

Zhang, L., Chiu, J., and Lin, J. C. (1998). Activation of human herpesvirus 8 (HHV-8) thymidine kinase (TK) TATAA-less promoter by HHV-8 ORF50 gene product is SP1 dependent. DNA Cell Biol. 17, 735-742.
Zhong, W., Wang, H., Herndier, B. and Ganem, D. (1996). Restricted expression of Kaposi sarcomaassociated herpesvirus (human herpesvirus 8) genes in Kaposi sarcoma. Proc. Natl. Acad. Sci. U.S.A 93, 6641-6646.

Zhu, F., Cusano, T., and Yuan, Y. (1999). Identification of the immediateearly transcripts of Kaposi's sarcoma-associated herpesvirus. J. Virol. 73, 5556-5567.

Ziegelbauer, J., Grundhoff, A., and Ganem, D. (2006). Exploring the DNA binding interactions of the Kaposi's sarcoma-associated herpesvirus lytic switch protein by selective amplification of bound sequences in vitro. J. Virol. 80 2958-2967.

Conflict of Interest Statement: The authors declare that the research was conducted in the absence of any commercial or financial relationships that could be construed as a potential conflict of interest.

Received: 08 December 2011; paper pending published: 27 December 2011; accepted: 18 January 2012; published online: 14 February 2012.

Citation: Guito J and Lukac DM (2012) KSHV Rta promoter specification and viral reactivation. Front. Microbio. 3:30. doi: 10.3389/fmicb.2012.00030

This article was submitted to Frontiers in Virology, a specialty of Frontiers in Microbiology.

Copyright (C) 2012 Guito and Lukac. This is an open-access article distributed under the terms of the Creative Commons Attribution Non Commercial License, which permits non-commercial use, distribution, and reproduction in other forums, provided the original authors and source are credited. 